\title{
Optimal Compensation with Hidden Action and Lump-Sum Payment in a Continuous-Time Model *
}

\author{
Jakša Cvitanić † Xuhu Wan ${ }^{\ddagger}$ and Jianfeng Zhang ${ }^{\S}$
}

May 16, 2008

\begin{abstract}
We consider a problem of finding optimal contracts in continuous time, when the agent's actions are unobservable by the principal, who pays the agent with a one-time payoff at the end of the contract. We fully solve the case of quadratic cost and separable utility, for general utility functions. The optimal contract is, in general, a nonlinear function of the final outcome only, while in the previously solved cases, for exponential and linear utility functions, the optimal contract is linear in the final output value. In a specific example we compute, the first-best principal's utility is infinite, while it becomes finite with hidden actions, which is increasing in value of the output. In the second part of the paper we formulate a general mathematical theory for the problem. We apply the stochastic maximum principle to give necessary conditions for optimal contracts. Sufficient conditions are hard to establish, but we suggest a way to check sufficiency using non-convex optimization.
\end{abstract}

*Research supported in part by NSF grants DMS 04-03575, DMS 06-31298 and DMS 06-31366, and through the Programme "GUEST" of the National Foundation For Science, Higher Education and Technological Development of the Republic of Croatia. We are solely responsible for any remaining errors, and the opinions, findings and conclusions or suggestions in this article do not necessarily reflect anyone's opinions but the authors'. We are grateful to the editor and the anonymous referees for helpful suggestions that improved the exposition of the paper.

${ }^{\dagger}$ Corresponding Author; Caltech, M/C 228-77, 1200 E. California Blvd. Pasadena, CA 91125. Ph: (626) 395-1784. E-mail: cvitanic@hss.caltech.edu

${ }^{\ddagger}$ Department of Information and Systems Management, HKUST Business School, Clear Water Bay, Kowloon, Hong Kong. Ph: +852 2358-7731. Fax: +852 2359-1908. E-mail: imwan@ust.hk.

$\S$ Department of Mathematics, USC, 3620 S Vermont Ave, MC 2532, Los Angeles, CA 90089-1113. Ph: (213) 740-9805. E-mail: jianfenz@usc.edu. 
Key Words and Phrases: Hidden action, moral hazard, second-best optimal contracts and incentives, Principal-Agent problems, stochastic maximum principle, Forward-Backward SDEs.

AMS (2000) Subject Classifications: 91B28, 93E20; JEL Classification: C61, C73, D82, J33, M52 


\section{Introduction}

This paper builds a general theory of optimal contracts between two parties in continuous time when the action of one party is not observable. In most existing continuous-time models of this type it is assumed that the parties have exponential utility functions, while we allow general utility functions.

In economics literature, these type of problems are known as Principal-Agent problems, with a principal who hires an agent to perform a certain task by controlling a given stochastic process. For example, shareholders hire a company executive whose effort has an effect on the company's stock price, or investors hire a portfolio manager to manage their wealth from investments. In a previous paper, Cvitanić, Wan and Zhang (2006), we study the case in which the actions of the agent are observed by the principal, the so-called first-best case. Here, we consider the second-best case of "hidden actions" or "moral hazard", in which the agent's control of the drift of the process is unobserved by the principal. Thus, it is harder (more expensive) for the principal to provide incentives to the agent in order to make her apply high effort. The seminal paper on this topic in the continuous-time framework is Holmstrom and Milgrom (1987), which showed that if both the principal and the agent have exponential utilities, then the optimal contract is linear. Schattler and Sung (1993) generalized those results using a dynamic programming and martingales approach of Stochastic Control Theory, and Sung (1995) showed that the linearity of the optimal contract still holds even if the agent can control the volatility, too. A nice survey of the literature is provided by Sung (2001).

Our model is similar to those papers, but we obtain further results also for non-exponential utility functions. We use a so-called weak formulation, meaning that the agent, with her actions, influences the distribution of the outcome process, or, more precisely its rate of return. This approach is first suggested by Mirrlees $(1974,1976)$, as explained nicely in Holmstrom (1979), p77. We will illustrate this approach first in a simple single-period model.

Different variations and applications of the problem are considered in Detemple, Govindaraj and Loewenstein (2001), Hugonnier and Kaniel (2008), Ou-Yang (2003), Sannikov (2004), DeMarzo and Sannikov (2006), Williams (2004), Sung (2005), Biais, Mariotti, Plantin and Rochet (2007). See also Müller (1998, 2000), and Hellwig and Schmidt (2003). The paper closest to ours is Williams (2004). That paper uses the stochastic maximum principle to characterize the optimal contract in the principal-agent problems with hidden information, in the case without volatility control. It focuses on the case of a continuously paid reward to the agent, while we study the case when the reward is paid once, at the end of the contract. Moreover, we prove our results from the scratch, thus getting them under weaker conditions. (Williams (2004) also deals with the so-called hidden states case, which we do 
not discuss here.) A very nice paper with a setting in which the payment to the agent is continuous is Sannikov (2007). That paper has a risk-neutral principal, and the agent has an arbitrary utility function.

While working on the general theory, we have been able to identify a special framework in which the problem is tractable even with general utility functions: under the assumption of a cost function which is quadratic in agent's effort and under a separable utility, we find an explicit solution for the contract payoff. To the best of our knowledge, this is the first time that the optimal second-best lump-sum contract payoff is explicitly described in a continuous-time contracting problem with hidden action, other than for exponential and linear utilities. The solution depends only on the final outcome (usually in a nonlinear way), and not on the history of the controlled process, the fact which was known before for exponential and linear utilities. The contract is determined from an equation which extends the classical Borch rule for marginal utilities of the first-best contract. It is an increasing function of the final value of the output, thus in the spirit of real-life contracts, such as call option contracts in executive compensation. While the optimal payoff is explicitly given as a solution to a nonlinear equation, the agent's optimal effort is obtained as a part of a solution to a "simple" Backward Stochastic Differential Equation (BSDE), which, in a Markovian framework, boils down to solving a linear parabolic PDE, a standard heat equation.

In a concrete example that we compute, with risk-neutral principal (linear utility) and risk-averse agent (logarithmic utility), the effort is an increasing function of the current value of the output. Interestingly, the first-best case for that example leads to infinite utility for the principal.

For the general theory, we provide a detailed discussion on how to check whether the necessary conditions we find are also sufficient. In particular, this is true for the separable utility case Our method is based on studying the agent's "value function", that is, her remaining expected utility process. In continuous-time stochastic control literature this method is known at least since Davis and Varaiya (1973). In dynamic Principal-Agent problems in discrete-time, it is used, among others, in Abreu, Pearce and Stacchetti (1986), (1990), and Phelan and Townsend (1991).

The theory for general non-separable utility functions is quite hard. If the necessary conditions determine a unique control process, then, if we proved existence of the optimal control, we would know that the necessary conditions are also sufficient. The existence of an optimal control is hard because, in general, the problem is not concave. It is related to the existence of a solution to Forward-Backward Stochastic Differential Equations (FBSDEs), possibly fully coupled. However, it is not known under which general conditions these equations have a solution. The FBSDEs theory is presented in the monograph Ma and Yong (1999). The method of the stochastic maximum principle that we use is covered in the book Yong and Zhou (1999). For other applications of the stochastic maximum principle in 
finance, see the recent book by Oksendal and Sulem (2004).

The paper is organized as follows: In Section 2 we set up the model. In Section 3 we analyze the tractable case of quadratic cost and separable utility. In Section 4, we find necessary conditions for the agent's problem and the principal's problem in general case. In Section 5 we discuss how to establish sufficiency and illustrate with the Holmstrom-Milgrom example. We conclude in Section 6 and provide longer proofs in Appendix.

\section{The setting}

\subsection{Model with symmetric information}

We first describe here the model appropriate when considering the full information, first-best case, in which the agent's actions are observed.

Let $\left\{W_{t}\right\}_{t \geq 0}$ be a standard Brownian Motion on a probability space $(\Omega, \mathcal{F}, P)$ and denote by $\mathbf{F}^{\mathbf{W}} \triangleq\left\{\mathcal{F}_{t}^{W}\right\}_{t \leq T}$ its augmented filtration on the interval $[0, T]$. The controlled state process, or output process is denoted $X=X^{u, v}$ and its dynamics are given by

$$
d X_{t}=u_{t} v_{t} d t+v_{t} d W_{t}, \quad X_{0}=x .
$$

Here for simplicity we assume all the processes are one-dimensional. The $\mathbf{F}^{W}$-adapted processes $u$ and/or $v$ may be controlled by an agent, who is hired by a principal to control the output process $X$.

For example, if $u$ is fixed and $v$ is controlled, process $X$ corresponds exactly to a value process of a portfolio which invests in a stock and a bank account, whose manager decides which amount $v_{t}$ of money to hold in the stock at time $t$, and keeps the rest in the bank account. The value $u_{t}$ corresponds then to the expected return rate of the stock above the risk-free rate. This is a well known and standard model in Financial Mathematics. If the manager can affect the mean return through her effort, for example by carefully choosing the assets in which to invest, then we can assume that $u_{t}$ is also chosen by the manager.

A more general model would be a standard general model of Stochastic Control Theory given by

$$
d X_{t}=b\left(t, X_{t}, u_{t}, v_{t}\right) d t+\sigma\left(t, X_{t}, v_{t}\right) d W_{t} .
$$

When $\sigma$ is nondegenerate, one can always set

$$
\tilde{v}_{t} \triangleq \sigma\left(t, X_{t}, v_{t}\right), \quad \tilde{u}_{t}=b\left(t, X_{t}, u_{t}, v_{t}\right) \sigma^{-1}\left(t, X_{t}, v_{t}\right) .
$$

Then (2.2) becomes (2.1). Moreover, under some monotonicity conditions on $b, \sigma$, one can write $u, v$ as functions of $(X, \tilde{u}, \tilde{v})$. In this sense, (2.1) and (2.2) are equivalent. We always consider models of type $(2.1)$. 
The "full information" case, in which the principal observes $X, u, v$ and thus also $W$, was studied in Cvitanić, Wan and Zhang (2006). In the so-called "hidden action" case, the principal can only observe the controlled process $X_{t}$, but not the underlying Brownian motion or the agent's control $u$ (so the agent's "action" $u_{t}$ is hidden to the principal). We present the appropriate model for this, second-best case, in the following section.

At time $T$, the principal gives the agent compensation in the form of a payoff $C_{T}=F(X$.), where $F: C[0, T] \rightarrow \mathbb{R}$ is a (deterministic) mapping. We note that since the principal is assumed to observe the process $X$ continuously, the volatility control $v$ can also be observed by the principal through the quadratic variation of $X$, under the assumption $v \geq 0$. Because he can verify what volatility has been used, for a given process $v$ the principal can design the payoff $F$ in order to induce the agent to implement it (or to "force" her to do so by harsh penalization). In this sense, we may consider $v$ as a control chosen by the principal instead of by the agent, as is usual in the literature when action is observed. We say that the pair $(F, v)$ is a contract.

The agent's problem is that, given a contract $(F, v)$, she needs to choose the control $u$ (over some admissible set which will be specified later) in order to maximize her utility

$$
V_{1}(F, v) \triangleq \sup _{u} V_{1}(u ; F, v) \triangleq \sup _{u} E\left[U_{1}\left(F\left(X^{u, v}\right), G_{T}^{u, v}\right)\right] .
$$

Here,

$$
G_{t}^{u, v} \triangleq \int_{0}^{t} g\left(s, X_{s}, u_{s}, v_{s}\right) d s
$$

is the accumulated cost of the agent, and with a slight abuse of notation we use $V_{1}$ both for the objective function and its maximum. We say a contract $(F, v)$ is implementable if there exists an effort process $u^{F, v}$ which maximizes the agent's utility given the contract, that is, it is such that

$$
V_{1}\left(u^{F, v} ; F, v\right)=V_{1}(F, v) .
$$

The principal maximizes her utility

$$
V_{2} \triangleq \max _{F, v, u^{F, v}} E\left[U_{2}\left(X_{T}^{u^{F, v}, v}-F\left(X^{u^{F, v}, v}\right)\right)\right],
$$

where the maximum is over all implementable contracts $(F, v)$ and corresponding agent's optimal efforts $u^{F, v}$, such that the following participation constraint or individual rationality (IR) constraint holds:

$$
V_{1}(F, v) \geq R
$$

Note that typically for a given contract $(F, v)$ the corresponding optimal effort $u^{F, v}$ of the agent will be unique, in which case the principal only maximizes over $(F, v)$.

Constant $R$ is the reservation utility of the agent and represents the value of the agent's outside opportunities, the minimum value she requires to accept the job. Functions $U_{1}$ and 
$U_{2}$ are utility functions of the agent and the principal. The typical cases studied in the literature are the separable utility case with $U_{1}(x, y)=U_{1}(x)-y$, and the non-separable case with $U_{1}(x, y)=U_{1}(x-y)$, where, with a slight abuse of notation, we use the same notation $U_{1}$ also for the function of one argument only. We could also have the same generality for $U_{2}$, but this makes less sense from the economics point of view.

Remark 2.1 A standard way to write the principal's problem is the one of looking for a socalled "constrained Pareto optimal" solution, which is such that no other contract satisfying the required constraints could make both parties better off. More precisely, the problem is written as

$$
\sup _{F, v, u}\left[E\left[U_{2}\left(X_{T}-F\right)\right]+\lambda V_{1}(u ; F, v)\right]
$$

subject to the constraint (2.4). When we restrict ourselves to implementable contracts, then this formulation is equivalent to our formulation above, because the choice of the "Lagrange multiplier" $\lambda$ is equivalent to the choice of the minimal agent's utility $R$, while the constraint (2.4) is absorbed into the principal's problem by setting the effort $u$ in the principal's problem (2.5) equal to $u^{F, v}$, that is, equal to an effort which the agent will choose optimally given a contract $(F, v)$. While our formulation is less standard for symmetric information problems, it is consistent with the approach we will use for solving the problem in the case of hidden action: we will first characterize the optimal effort of the agent for a given contract, and then solve the principal's problem analogous to (2.5).

Remark 2.2 Our approach also works for the framework in which the output process $X=$ $\left(X^{1}, \ldots, X^{d}\right), d \geq 1$ is a $d$-dimensional vector. In this case effort $u$ and Brownian motion $W$ would also be $d$-dimensional vectors, while $v$ would be a $d \times d$ matrix. The principal's utility may take the general form $U_{2}\left(X_{T}, C_{T}\right)$, or a special form $U_{2}\left(\sum_{i} X_{T}^{i}-C_{T}\right)$ as in Holmstrom and Milgrom (1987). By observing $X$ continuously, the principal also observes its quadratic variation matrix $v v^{*}$, where $v^{*}$ denotes the transpose of $v$. Notice that the principal cannot observe $v$ directly. If we assume further that, given $v v^{*}$, the particular choice of $v$ does not change the value of the cost $G_{T}$, then the principal essentially "observes" $v$, and one can extend all the results in this paper without substantial difficulty.

\subsection{Model with hidden action}

For the origins, importance and numerous applications of the discrete-time Principal-Agent theory with hidden action (and more), we refer the reader to the excellent book Bolton and Dewatripont (2005). The original motivation behind continuous-time models in the seminal paper Holmstrom and Milgrom (1987) was to show that if the agent has a rich strategy space, then she can undermine complicated contracts, and the principal is forced to use 
simple contracts, as is frequently the case in practice. Before their paper, there was a gap between the theory, in which complex contracts were optimal, and practice, in which often relatively simple contracts are observed. They also show that their continuous-time model in which the drift is controlled, but not the volatility, can be obtained as a limit of a model in which the outcomes have multinomial distribution, and in which the agent chooses the probabilities of the outcomes, while the possible outcomes values are fixed. We essentially adopt Holmstrom and Milgrom continuous-time model for the underlying dynamics, as do most other existing papers in continuous time.

\subsubsection{A single-period model with hidden action}

In order to make it easier to understand our continuous-time model, we first present a simple problem in a single-period model. This is a familiar model, and can be found, for example, in the book Bolton and Dewatripont (2005). This modeling approach, of the action determining the distribution of the output, was originally suggested by Mirrlees $(1974,1976)$ and extended in Homstrom (1979). It was driven partly by tractability, as Mirrlees (1974, 1976) has shown that without this approach even simple problems may be hard or not have a solution.

In this model we describe the contract which is optimal among linear contracts, while in the analogues continuous-time model the same linear contract turns out to be optimal even if we allow general contracts. This is an advantage of continuous-time, as discovered first in Holmstrom and Milgrom (1987).

The agent picks the distribution of the final output $X_{1}$ by her action, unobserved by the principal. We assume that under a fixed probability $P=P^{0}$, we have

$$
X_{1}=X_{0}+v B_{1}
$$

where $v$ is fixed and $B_{1}$ is a random variable with a standard normal distribution. By applying action $u$, the probability $P$ changes to $P^{u}$, under which the distribution of $B_{1}$ is normal with mean $u$ and variance one. Therefor, under $P^{u}, X_{1}$ has mean $u v$.

We consider only contracts which are linear in $X_{1}$, or, equivalently, in $B_{1}$ :

$$
C_{1}=k_{0}+k_{1} B_{1}
$$

We assume a quadratic cost function, $g(u)=u^{2} / 2$, and exponential utility functions. Denoting by $E^{u}$ the expectation operator under probability $P^{u}$, we define the agent's problem to be the minimization of

$$
E^{u}\left[e^{-\gamma_{1}\left(k_{0}+k_{1} B_{1}-u^{2} / 2\right)}\right]=e^{-\gamma_{1}\left(k_{0}-u^{2} / 2+k_{1} u+\frac{1}{2} k_{1}^{2} \gamma_{1}\right)}
$$

where we used the fact that

$$
E^{u}\left[e^{k B_{1}}\right]=e^{k u+\frac{1}{2} k^{2}}
$$


Hence, the optimal action $u$ is

$$
u=k_{1}
$$

We now describe a method which will also use in the continuous-time case. We suppose that the principal decides to provide the agent with a contract payoff $C_{1}$ which results in (optimal) expected utility of $R$ to the agent. This means that, using (2.7) and (2.8),

$$
R=-\frac{1}{\gamma_{1}} E^{u}\left[e^{-\gamma_{1}\left(C_{1}-u^{2} / 2\right)}\right]=-\frac{1}{\gamma_{1}} e^{-\gamma_{1}\left(k_{0}-\frac{1}{2} u^{2}-\frac{1}{2} \gamma_{1} u^{2}\right)}
$$

Computing $e^{-\gamma_{1} k_{0}}$ from this and using $C_{1}=k_{0}+u B_{1}$, we can write

$$
-\frac{1}{\gamma_{1}} e^{-\gamma_{1} C_{1}}=R e^{-\gamma_{1}\left(\frac{1}{2}\left[\gamma_{1}+1\right] u^{2}+u B_{1}\right)}
$$

Thus, we get a representation of the contract payoff in terms of the agent's "promised" utility $R$ and the source of uncertainty $B_{1}$, something we will find helpful later on, too. Denoting the principal's risk aversion with $\gamma_{2}$, using $e^{\gamma_{2} C_{1}}=\left(e^{-\gamma_{1} C_{1}}\right)^{-\gamma_{2} / \gamma_{1}}, X_{1}=X_{0}+v B_{1}$ and (2.10), we can write the principal's expected utility as

$$
E^{u}\left[U_{P}\left(X_{1}-C_{1}\right)\right]=-\frac{1}{\gamma_{2}}(R)^{-\gamma_{2} / \gamma_{1}} E^{u}\left[e^{-\gamma_{2}\left(X_{0}+v B_{1}-\frac{1}{2}\left[\gamma_{1}+1\right] u^{2}-u B_{1}\right)}\right]
$$

which can be computed as

$$
-\frac{1}{\gamma_{2}} R^{-\gamma_{2} / \gamma_{1}} e^{-\gamma_{2}\left(X_{0}-\gamma_{2} v^{2} / 2+\left(1+\gamma_{2}\right) u v-\frac{1}{2} u^{2}\left(1+\gamma_{1}+\gamma_{2}\right)\right)}
$$

Maximizing over $u$, we get the optimal $u$ as

$$
u=v \frac{1+\gamma_{2}}{1+\gamma_{1}+\gamma_{2}}
$$

If the principal could choose $v$, he would optimize the above expression over $v$, too.

If there was symmetric information, and the model was

$$
X_{1}=X_{0}+a v+v B_{1}
$$

(under a single, fixed probability $P$ ), in a similar way it can be computed that the optimal action is $u=v$, and that the contract is of the form

$$
C_{1}=c+\frac{\gamma_{2}}{\gamma_{1}+\gamma_{2}} X_{1}
$$

We see that the "sensitivity" $\frac{\gamma_{2}}{\gamma_{1}+\gamma_{2}}$ of the first-best contract is less than the sensitivity $k_{1} / v=\frac{1+\gamma_{2}}{1+\gamma_{1}+\gamma_{2}}$ of the second-best contract. This is not surprising - when the action is unobservable the principal is forced to try to induce more effort by offering higher incentives.

As mentioned above, when we illustrate our theory with the Holmstrom-Milgrom (1987) problem in continuous time, we will see that the above second-best contract is actually optimal among all contracts, linear or not. 


\subsubsection{A continuous-time model with hidden action}

Similarly to our single-period model, Holmstrom-Milgrom (1987) assume that the agent is choosing a probability measure under which the output process evolves (as can be seen in particular in their proof of Theorem 6 in the appendix of that paper). Schattler and Sung (1993) make this approach precise and rigorous, based on the so-called weak formulation and a martingale approach to stochastic control. We adopt the same formulation, and describe it in more detail next.

Let $B$ be a standard Brownian motion under some probability space with probability measure $Q$, and $\mathbf{F}^{B}=\left\{\mathcal{F}_{t}^{B}\right\}_{0 \leq t \leq T}$ be the filtration on $[0, T]$ generated by $B$. For any $\mathbf{F}^{B}$-adapted square integrable process $v>0$, let

$$
X_{t} \triangleq x+\int_{0}^{t} v_{s} d B_{s}
$$

Then $v_{t}$ is a functional of $X, v_{t}=\tilde{v}_{t}(X$.$) and obviously it holds that$

$$
\mathcal{F}_{t}^{X}=\mathcal{F}_{t}^{B}, \quad \forall t
$$

Moreover, effort process $u$ is assumed to be a functional $\tilde{u}_{t}$ of $X$. Given such $\tilde{u}$, we define

$$
u_{t} \triangleq \tilde{u}_{t}(X .) ; \quad B_{t}^{u} \triangleq B_{t}-\int_{0}^{t} u_{s} d s ; \quad M_{t}^{u} \triangleq \exp \left(\int_{0}^{t} u_{s} d B_{s}-\frac{1}{2} \int_{0}^{t}\left|u_{s}\right|^{2} d s\right) ;
$$

and a new probability measure $Q^{u}$ by

$$
\frac{d Q^{u}}{d Q} \triangleq M_{T}^{u}
$$

Then we know by Girsanov Theorem that, under certain conditions, $B^{u}$ is a $Q^{u}$-Brownian motion and

$$
d X_{t}=v_{t} d B_{t}=\left(\tilde{u}_{t} \tilde{v}_{t}\right)(X .) d t+\tilde{v}_{t}(X .) d B_{t}^{u} .
$$

That is, in the language of Stochastic Analysis, the triple $\left(X, B^{u}, P^{u}\right)$ is a weak solution to the SDE

$$
d X_{t}=\left(\tilde{u}_{t} \tilde{v}_{t}\right)(X .) d t+\tilde{v}_{t}(X .) d W_{t} .
$$

Compared to (2.1), we note that in the weak formulation we consider functionals $(\tilde{u}, \tilde{v})$ as controls. Accordingly, we consider $(F, \tilde{v})$ as a contract offered by the principal. The choice of $\tilde{u}$ corresponds to the choice of probability measure $P^{u}$, thus to the choice of the distribution of process $X$. It is also well known that this is the only way to vary probability measures in Brownian models, while keeping them equivalent (i.e., having the same null sets).

For any contract payoff $C_{T} \in \mathcal{F}_{T}^{B}$, there exists some functional $F$ such that $C_{T}=F(X$.). Thus, a contract $(F, \tilde{v})$ is equivalent to a random variable $C_{T} \in \mathcal{F}_{T}^{B}$ and a process $v \in \mathbf{F}^{B}$. 
Also, an action $\tilde{u}$ is equivalent to a process $u \in \mathbf{F}^{B}$. For simplicity, in the following we abuse the notation by writing $u_{t}=\tilde{u}_{t}(X$.$) and v_{t}=\tilde{v}_{t}(X$.) when there is no danger of confusion.

Now given a contract $C_{T} \in \mathcal{F}_{T}^{B}$ and $v \in \mathbf{F}^{B}$, the agent's problem is to find an optimal control $u^{C_{T}, v} \in \mathbf{F}^{B}$ such that

$$
V_{1}\left(u^{C_{T}, v} ; C_{T}, v\right)=V_{1}\left(C_{T}, v\right) \triangleq \sup _{u} V_{1}\left(u ; C_{T}, v\right)
$$

where, recalling (2.3),

$$
V_{1}\left(u ; C_{T}, v\right) \triangleq E^{Q^{u}}\left\{U_{1}\left(C_{T}, G_{T}\right)\right\}=E^{Q}\left\{M_{T}^{u} U_{1}\left(C_{T}, G_{T}\right)\right\} .
$$

For simplicity from now on we denote $E \triangleq E^{Q}$ and $E^{u} \triangleq E^{Q^{u}}$. The principal's problem is to find optimal $\left(C_{T}^{*}, v^{*}\right)$ such that

$$
V_{2}\left(C_{T}^{*}, v^{*}\right)=V_{2} \triangleq \sup _{C_{T}, v, u^{C}, v} V_{2}\left(u^{C_{T}, v} ; C_{T}, v\right),
$$

where

$$
V_{2}\left(u ; C_{T}, v\right) \triangleq E^{u}\left\{U_{2}\left(X_{T}-C_{T}\right)\right\}=E\left\{M_{T}^{u} U_{2}\left(X_{T}-C_{T}\right)\right\} .
$$

We see from this last expression that, indeed, the choice of $u$ is really the choice of $M^{u}$ and hence the choice of the probability measure, that is, the choice of distribution of the output $X$.

As usual in contract theory, we assume that when the agent is indifferent between two actions, she will choose the one better for the principal.

Remark 2.3 (i) The agent chooses her action based on the output value $X$ which is observable to the principal. However, although $u$ is $\mathbf{F}^{X}$-adapted, the principal does not know $\tilde{u}$, and hence does not know the value of $B^{u}$ either.

(ii) Mathematically, the strong formulation we used in Section 2.1 and the weak formulation of this section are in general not equivalent, due to the different requirements on the measurability of the agent's control $u$. In Section 2.1, $u$ is an $\mathbf{F}^{W}$-adapted process, and thus $\mathbf{F}^{X^{u}}$ may be smaller than $\mathbf{F}^{W}$. In contrast, in the weak formulation here, $u$ is $\mathbf{F}^{X}$-adapted, and thus $\mathbf{F}^{B^{u}}$ may be smaller than $\mathbf{F}^{X}$.

(iii) If we restrict $u$ to a smaller admissible set, say to those such that $\mathbf{F}^{X^{u}}=\mathbf{F}^{W}$ in the strong formulation and those such that $\mathbf{F}^{X}=\mathbf{F}^{B^{u}}$ in the weak formulation, then the two formulations are equivalent. This constraint, however, may make the calculations in the general framework very difficult.

(iv) However, in the solvable examples existing in the literature, it turns out that, for the optimal $u$, the two filtrations are equal (e.g., the solution $u$ in the strong formulation is a feedback control, or even deterministic); see below for more comments. 
(iv) In the strong formulation, one has to distinguish the optimal contract $F$ and its realized optimal value $C_{T}=F(X$.) (see, e.g. Cvitanić, Wan and Zhang (2006)). In the weak formulation, since, given the outcome $\omega \in \Omega$, the output value of $X$ is fixed (independent of $u$ ), the random variable $C_{T}$ can be understood as the contract, and we do so in this paper.

We note that often in the literature, for tractability reasons the weak formulation is used for the agent's problem and the strong formulation for the principal's problem. However, there is a subtle measurability issue, as pointed out in part (ii) of the above remark, More precisely, on one hand, the optimal action $u^{F, v}$ obtained from the agent's problem by using the weak formulation may not be in the admissible set under the strong formulation (if $\mathbf{F}^{B^{u}}$ is strictly smaller than $\mathbf{F}^{X}$ ); on the other hand, given a principal's target action $u$ (see $\S 4.2 .2$ ) in the strong formulation, it is not always possible to obtain it as an optimal solution of the agent's problem in the weak formulation, as it may not be even implementable. In this paper we are able to develop a general theory using the weak formulation for both the agent's problem and the principal's problem, avoiding potential inconsistencies. On the other hand, as mentioned in part (iv) of the above remark, in all the solvable examples in the literature it turns out that the optimal, for the strong formulation, effort $u$ is a functional of output $X$ only (and not of Brownian Motion $W$ ). If that is the case, one may use the strong formulation for the principal's problem without loss of generality.

We now present a special case which can be solved more or less straightforwardly, and it is probably the most interesting part of the paper for economic applications. Later, we present a more fully developed mathematical theory, which, unfortunately does not lead to general existence results, or additional solvable examples, but it does provide necessary conditions for optimality.

\section{Special case: Separable utility with fixed volatility $v$ and quadratic cost $g$}

The model we present here is quite general in the choice of the utility functions, and thus could be of use in many economic applications. The solution is explicit in the characterization of the optimal contract payoff $C_{T}$, via a nonlinear deterministic equation. It is in general semi-explicit in terms of the optimal effort $u$, as it boils down to solving a linear BSDE (not FBSDE!). To the best of our knowledge, this is the first explicit description of a solution to a continuous-time Principal-Agent problem with hidden action and lump-sum payment, other than the case of exponential and linear utility functions. Moreover, as in those two cases, the optimal contract is still a function only of the final outcome $X_{T}$, and not of the history of the output process $X$, but unlike those two cases, the dependence on $X_{T}$ is nonlinear in general. 
We assume the process $v$ is fixed; the agent takes separable utility $U_{1}\left(C_{T}\right)-G_{T}$; and the cost function is

$$
g(t, x, u, v)=u^{2} / 2 .
$$

First we adopt standard assumptions for utility functions, which are assumed throughout this section.

Assumption $3.1 U_{1}, U_{2}$ are twice differentiable such that $U_{i}^{\prime}>0, U_{i}^{\prime \prime} \leq 0, i=1,2$.

We now specify the technical conditions $u$ and $C_{T}$ should satisfy. Roughly speaking, we need enough integrability so that calculations in the remaining of the section can go through.

Definition 3.1 The set $\mathcal{A}_{1}$ of admissible effort processes $u$ is the space of $\mathbf{F}^{B}$-adapted processes $u$ such that

(i) $P\left(\int_{0}^{T}\left|u_{t}\right|^{2} d t<\infty\right)=1$;

(ii) $E\left\{\left|M_{T}^{u}\right|^{4}\right\}<\infty$.

We note that any $u \in \mathcal{A}_{1}$ satisfies the Novikov condition and thus the Girsanov Theorem can be applied, see (7.1) at below.

Definition 3.2 The set $\mathcal{A}_{2}$ of admissible contracts is the space of $\mathcal{F}_{T}^{B}$-measurable $C_{T}$ such that

(i) $E\left\{\left|U_{1}\left(C_{T}\right)\right|^{4}+e^{4 U_{1}\left(C_{T}\right)}\right\}<\infty$.

(ii) $E\left\{\left|U_{2}\left(X_{T}-C_{T}\right)\right|^{2}+e^{U_{1}\left(C_{T}\right)}\left|U_{2}\left(X_{T}-C_{T}\right)\right|\right\}<\infty$.

\subsection{Agent's problem}

First we establish a simple technical lemma for a linear BSDE.

Lemma 3.1 Assume $u \in \mathbf{F}^{B}$, Girsanov Theorem holds true for $\left(B^{u}, Q^{u}\right)$, and $E\left\{\left|M_{T}^{u}\right|^{2}\right\}<$ $\infty$. Then for any $\xi \in \mathcal{F}_{T}^{B}$ such that $E^{u}\left\{|\xi|^{2}\right\}<\infty$, there exists a unique $Q^{u}$-square integrable pair $(Y, Z) \in \mathbf{F}^{B}$ such that

$$
Y_{t}=\xi-\int_{t}^{T} Z_{s} d B_{s}^{u}
$$

Obviously $Y_{t}=E_{t}^{u}\{\xi\}$, and uniqueness also follows immediately. But in general $\mathbf{F}^{B^{u}} \neq$ $\mathbf{F}^{B}$, so we cannot apply the standard Martingale Representation Theorem directly to obtain $Z$. Nevertheless, the result follows from an extended Martingale Representation Theorem, see Fujisaki, Kallianpur and Kunita (1972), and for completeness we provide a proof in Appendix. We now solve the agent's problem. 
Theorem 3.1 For any contract $C_{T} \in \mathcal{A}_{2}$, the optimal effort $\hat{u} \in \mathcal{A}_{1}$ for the agent is obtained by solving the BSDE

$$
\bar{Y}_{t}=E_{t}\left[e^{U_{1}\left(C_{T}\right)}\right]=e^{U_{1}\left(C_{T}\right)}-\int_{t}^{T} \hat{u}_{s} \bar{Y}_{s} d B_{s}
$$

Moreover, the agent's optimal expected utility is given by

$$
V_{1}=\log \bar{Y}_{0}=\log E\left[e^{U_{1}\left(C_{T}\right)}\right] .
$$

Remark 3.1 (i) We can see from the proof below that

$$
\log \bar{Y}_{t}=U_{1}\left(C_{T}\right)-\frac{1}{2} \int_{t}^{T} \hat{u}_{s}^{2} d s-\int_{t}^{T} \hat{u}_{s} d B_{s}^{\hat{u}}
$$

represents the remaining expected utility of the agent when she behaves optimally. Thus, the optimal $\hat{u}$ is chosen so that the remaining expected utility is increasing by the reduction in the remaining cost until it reaches $U_{1}\left(C_{T}\right)$, and there is additional mean zero term $\int_{t}^{T} \hat{u}_{s} d B_{s}^{\hat{u}}$. Actually, the fact that the integrand of this term is equal to $\hat{u}$ distinguishes the optimal effort from non-optimal efforts.

(ii) One of the main insights of Holmstrom-Milgrom (1987) and Schattler and Sung (1993) is the representation of the optimal payoff $C_{T}$ in terms of the agent's optimal effort $\hat{u}$ and her utility certainty equivalent. That representation corresponds in our model of this section to equation (3.4), after a transformation (the certainty equivalent is $U_{1}^{-1}\left(\log \bar{Y}_{t}\right)$ ). For our case of separable utilities it is more convenient to work with the remaining utility as in (3.4) than with the certainty equivalent.

(iii) In the language of option pricing theory finding $\hat{u}$ is equivalent to finding a replicating portfolio for the option with payoff $e^{U_{1}\left(C_{T}\right)}$. Numerous methods have been developed to compute such an object, sometimes analytically, otherwise numerically. Let us comment on this in more detail. As we will see below, the optimal contract (when exists) is going to be a deterministic function of $X_{T}$, say $C_{T}=c\left(X_{T}\right)$. Assume $v_{t}=v\left(t, X_{t}\right)$ is a deterministic function of $X$ and $t$ and thus $X$ is Markovian. Then the solution to the BSDE (3.2) is a function of $X_{t}$, that is, $\bar{Y}_{t}=F\left(t, X_{t}\right)$ and

$$
d F\left(t, X_{t}\right)=\left[F_{t}+\frac{1}{2} F_{x x} v_{t}^{2}\right] d t+F_{x} v_{t} d B_{t}
$$

Since $\bar{Y}$ is a martingale, the $d t$ term has to be zero, so that $F$ satisfies the PDE

$$
\left\{\begin{array}{l}
F_{t}(t, x)+\frac{1}{2} v^{2}(t, x) F_{x x}(t, x)=0 \\
F(T, x)=e^{U_{1}(c(x))}
\end{array}\right.
$$

and

$$
u_{t}=u\left(t, X_{t}\right)=\frac{v\left(t, X_{t}\right) F_{x}\left(t, X_{t}\right)}{F\left(t, X_{t}\right)}
$$


Proof of the theorem: We first claim that the BSDE (3.2) is well-posed and that $\hat{u}$ determined by (3.2) is indeed in $\mathcal{A}_{1}$. The proof is technical and thus is postponed to the Appendix.

We now show that $\hat{u}$ is optimal for the agent's problem. For any $u \in \mathcal{A}_{1}$, as is standard in this type of stochastic control problems (see Schattler and Sung 1993, for example) and, in particular, in dynamic principal-agent problems, we consider the remaining utility of the agent at time $t$

$$
W_{t}^{A, u}=E_{t}^{u}\left[U_{1}\left(C_{T}\right)-\frac{1}{2} \int_{t}^{T} u_{s}^{2} d s\right]
$$

Then $W_{t}^{A, u}-\frac{1}{2} \int_{0}^{t} u_{s}^{2} d s$ is a $Q^{u}$-martingale, so by Lemma 3.1 there exists an $\mathbf{F}^{B}$-adapted process $Z^{A, u}$ such that

$$
W_{t}^{A, u}-\frac{1}{2} \int_{0}^{t} u_{s}^{2} d s=U_{1}\left(C_{T}\right)-\frac{1}{2} \int_{0}^{T} u_{s}^{2} d s-\int_{t}^{T} Z_{s}^{A, u} d B_{s}^{u} .
$$

Then, switching from $B^{u}$ to $B$, we have

$$
W_{t}^{A, u}=U_{1}\left(C_{T}\right)+\int_{t}^{T}\left[u_{s} Z_{s}^{A, u}-\frac{1}{2} u_{s}^{2}\right] d s-\int_{t}^{T} Z_{s}^{A, u} d B_{s}
$$

Note that $W_{0}^{A, u}=E^{u}\left[U_{1}\left(C_{T}\right)-\frac{1}{2} \int_{0}^{T} u_{s}^{2} d s\right]$, is the agent's utility, given action $u$. On the other hand, for $\hat{u}$ from the theorem, using Itô's rule and (3.2), we get

$$
\log \bar{Y}_{t}=U_{1}\left(C_{T}\right)+\frac{1}{2} \int_{t}^{T} \hat{u}_{s}^{2} d s-\int_{t}^{T} \hat{u}_{s} d B_{s} .
$$

Thus, $\log \bar{Y}_{0}=E\left[\log \bar{Y}_{0}\right]=W_{0}^{A, \hat{u}}$ is the agent's utility if she chooses action $\hat{u}$. Notice that

$$
\begin{aligned}
W_{0}^{A, \hat{u}}-W_{0}^{A, u} & =\int_{0}^{T}\left[\frac{1}{2}\left[\left|\hat{u}_{t}\right|^{2}+\left|u_{t}\right|^{2}\right]-u_{t} Z_{t}^{A, u}\right] d t+\int_{0}^{T}\left[Z_{t}^{A, u}-\hat{u}_{t}\right] d B_{t} \\
& \geq \int_{0}^{T}\left[\hat{u}_{t} u_{t}-u_{t} Z_{t}^{A, u}\right] d t+\int_{0}^{T}\left[Z_{t}^{A, u}-\hat{u}_{t}\right] d B_{t} \\
& =\int_{0}^{T}\left[Z_{t}^{A, u}-\hat{u}_{t}\right] d B_{t}^{u} .
\end{aligned}
$$

The equality holds if and only if $u=\hat{u}$. Note that $E^{u}\left\{\int_{0}^{T}\left|Z_{t}^{A, u}\right|^{2} d t\right\}<\infty$, and

$$
E^{u}\left\{\int_{0}^{T}\left|\hat{u}_{t}\right|^{2} d t\right\}=E\left\{M_{T}^{u} \int_{0}^{T}\left|\hat{u}_{t}\right|^{2} d t\right\} \leq C E\left\{\left|M_{T}^{u}\right|^{2}+\left(\int_{0}^{T}\left|\hat{u}_{t}\right|^{2} d t\right)^{2}\right\}<\infty,
$$

thanks to (7.1) at below. Then

$$
E^{u}\left\{\int_{0}^{T}\left[Z_{t}^{A, u}-\hat{u}_{t}\right]^{2} d t\right\}<\infty .
$$

Taking expected values under $Q^{u}$ in (3.8) we get $W_{0}^{A, \hat{u}} \geq W_{0}^{A, u}$, with equality if and only if $u=\hat{u}$. 


\subsection{Principal's problem}

Since, given a contract $C_{T} \in \mathcal{A}_{2}$, the agent's optimal effort $\hat{u}=\hat{u}\left(C_{T}\right)$ from Theorem 3.1 is unique, the principal's optimization problem can be written as

$$
\sup _{C_{T}} E^{\hat{u}\left(C_{T}\right)}\left[U_{2}\left(X_{T}-C_{T}\right)\right]
$$

under the constraint (2.6).

Note now that by solving the linear equation (3.2), we get, denoting henceforth $\hat{u}=u$,

$$
\bar{Y}_{t}=\bar{Y}_{0} e^{\int_{0}^{t} u_{s} d B_{s}-\frac{1}{2} \int_{0}^{t} u_{s}^{2} d s}
$$

which, together with (2.13) and (3.3), gives the following crucial fact

$$
U_{1}\left(C_{T}\right)=\log \bar{Y}_{T}=V_{1}+\log \left(M_{T}^{u}\right)
$$

This turns out to be exactly the reason why this problem is tractable: the fact that for the optimal agent's effort $u$, we have

$$
M_{T}^{u}=e^{-V_{1}} e^{U_{1}\left(C_{T}\right)}
$$

In other words, the choice of the probability measure corresponding to the optimal action $u$ has an explicit functional relation with the promised payoff $C_{T}$.

When we use this expression, and recall that $E^{u}[X]=E\left[M_{T}^{u} X\right]$, we can rewrite the principal's problem as

$$
\sup _{C_{T}, V_{1}} e^{-V_{1}} E\left\{e^{U_{1}\left(C_{T}\right)}\left[U_{2}\left(X_{T}-C_{T}\right)+\lambda\right]\right\},
$$

where $\lambda$ is a Lagrange multiplier for the IR constraint $E\left[e^{U_{1}\left(C_{T}\right)}\right]=e^{V_{1}}$ (see (3.3)).

As usual in hidden action problems, we see that the principal will give the smallest possible utility to the agent,

$$
V_{1}=R
$$

Moreover, we get

Proposition 3.1 Assume that the contract $C_{T}$ is required to satisfy

$$
L \leq C_{T} \leq H
$$

for some $\mathcal{F}_{T}$-measurable random variables $L, H$, which may take infinite values. If, with probability one, there exists a finite value $\hat{C}_{T}^{\lambda}(\omega) \in[L(\omega), H(\omega)]$ that maximizes

$$
e^{U_{1}\left(C_{T}\right)}\left[U_{2}\left(X_{T}-C_{T}\right)+\lambda\right]
$$

and $\lambda$ can be found so that

$$
E\left[e^{U_{1}\left(\hat{C}_{T}^{\lambda}\right)}\right]=e^{R}
$$

then $\hat{C}_{T}^{\lambda}$ is the optimal contract. 
Since (3.13) is considered $\omega$ by $\omega$, we reduce the problem to a one-variable deterministic optimization problem.

Remark 3.2 In this remark we assume that there is an interior solution when maximizing (3.13).

(i) The first order condition for maximizing (3.13) can be written as

$$
\frac{U_{2}^{\prime}\left(X_{T}-C_{T}\right)}{U_{1}^{\prime}\left(C_{T}\right)}=\lambda+U_{2}\left(X_{T}-C_{T}\right)
$$

This is a generalization of the classical Borch rule for risk-sharing in the first-best (full information) case:

$$
\frac{U_{2}^{\prime}\left(X_{T}-C_{T}\right)}{U_{1}^{\prime}\left(C_{T}\right)}=\lambda .
$$

The difference is the last term in (3.14): the ratio of marginal utilities of the agent and the principal is no longer constant, but a constant plus utility of the principal. Increase in global utility of the principal also makes him happier at the margin, relative to the agent, and decrease in global utility makes him less happy at the margin. This will tend to make the contract "more nonlinear" than in the first-best case. For example, if both utility functions are exponential, and we require $C_{T} \geq L>-\infty$ (for technical reasons), it is easy to check from (3.15) that the first-best contract $C_{T}$ will be linear in $X_{T}$ for $C_{T}>L$. On the other hand, as can be seen from (3.14), the second-best contract will be nonlinear.

(ii) Note that the optimal contract is a function of the final value $X_{T}$ only. In the previous continuous-time literature, only the cases of exponential (non-separable) utility functions and linear utility functions have been solved explicitly, leading to linear contracts. It's been stated that in the case of general utilities the optimal contract may depend on the history of the process $X$. However, this is not the case in the special framework of this section.

(iii) The first order condition can be solved for the optimal contract $C_{T}=C_{T}\left(X_{T}\right)$ as a function of $X_{T}$, and we can find, omitting the functions arguments, that

$$
\frac{\partial}{\partial X_{T}} C_{T}=1-\frac{U_{2}^{\prime} U_{1}^{\prime \prime}}{U_{2}^{\prime \prime} U_{1}^{\prime}+U_{2}^{\prime} U_{1}^{\prime \prime}-U_{2}^{\prime}\left(U_{1}^{\prime}\right)^{2}} .
$$

Thus, the contract is a non-decreasing function of $X_{T}$, and its slope with respect to $X_{T}$ is not higher than one.

In the first-best case of (3.15), we have

$$
\frac{\partial}{\partial X_{T}} C_{T}=1-\frac{U_{2}^{\prime} U_{1}^{\prime \prime}}{U_{2}^{\prime \prime} U_{1}^{\prime}+U_{2}^{\prime} U_{1}^{\prime \prime}} .
$$


We see that the sensitivity of the contract is higher in the second-best case, because more incentives are needed to induce the agent to provide optimal effort when the effort is hidden. The term which causes the increase in the slope of the contract is $U_{2}^{\prime}\left(U_{1}^{\prime}\right)^{2}$ in the denominator. We see that this term is dominated by the agent's marginal utility, but it also depends on the principal's marginal utility. Higher marginal utility for either party causes the slope of the contract to increase relative to the first-best case.

There is also an alternative way to formulate the principal's problem, as optimizing over effort $u$, or probability measures $Q^{u}$, which we present next. From $(3.10)$, with $V_{1}=R$, the principal's problem is

$$
\sup _{u} E\left[G\left(M_{T}^{u}\right)\right] \triangleq \sup _{u} E\left[M_{T}^{u} U_{2}\left(X_{T}-J_{1}\left(R+\log \left(M_{T}^{u}\right)\right)\right)\right]
$$

Here, $J_{1} \triangleq U_{1}^{-1}$ and $G$ is a random function on positive real numbers, defined by

$$
G(x) \triangleq x U_{2}\left(X_{T}-J_{1}(R+\log (x))\right.
$$

It is straightforward to compute that

$$
G^{\prime \prime}(x)<0
$$

so that $G$ is a strictly concave function, for every fixed $X_{T}(\omega)$.

We define the dual function, for $y>0$,

$$
\tilde{G}(y)=\max _{x \geq 0}[G(x)-x y] .
$$

The maximum is attained at

$$
\hat{x}=\left[\left(G^{\prime}\right)^{-1}(y)\right]^{+} .
$$

Thus, we get the following upper bound on the principal's problem, for any constant $\lambda>0$ :

$$
E\left[G\left(M_{T}^{u}\right)\right] \leq E[\tilde{G}(\lambda)]+\lambda E\left[M_{T}^{u}\right]=E[\tilde{G}(\lambda)]+\lambda .
$$

The upper bound will be attained if

$$
M_{T}^{u}=\left(G^{\prime}\right)^{-1}(\lambda)
$$

and $\lambda$ is chosen such that

$$
E\left[\left(G^{\prime}\right)^{-1}(\lambda)\right]=1
$$

This leads to the following alternative to the Proposition 3.1 
Proposition 3.2 Assume (for simplicity) that there are no required bounds on the contract $C_{T}$. If, with probability one, $\left(G^{\prime}\right)^{-1}(\lambda)>0$ for $\lambda$ for which $E\left[\left(G^{\prime}\right)^{-1}(\lambda)\right]=1$, then the optimal effort $u$ satisfies the Backward SDE

$$
M_{t}^{u}=\left(G^{\prime}\right)^{-1}(\lambda)-\int_{t}^{T} u_{s} M_{s}^{u} d B_{s}
$$

(assuming a solution exists). Moreover, we have

$$
\left(G^{\prime}\right)^{-1}(\lambda)=e^{-R} e^{\Phi\left(X_{T}, \lambda\right)}
$$

where $C_{T}=\Phi\left(X_{T}, \lambda\right)$ is a solution to (3.14), and it is the optimal payoff.

Proof: By the above upper bound, $M_{T}^{u}=\left(G^{\prime}\right)^{-1}(\lambda)$ is optimal for the principal's problem, and the BSDE from the proposition is the consequence of the dynamics of the process $M_{t}$. Moreover, we have

$$
G^{\prime}\left(e^{-R} e^{\Phi\left(X_{T}, \lambda\right)}\right)=U_{2}\left(X_{T}-J_{1}\left(\Phi\left(X_{T}, \lambda\right)\right)-U_{2}^{\prime}\left(X_{T}-J_{1}\left(\Phi\left(X_{T}, \lambda\right)\right)\right) J_{1}^{\prime}\left(\Phi\left(X_{T}, \lambda\right)\right)=\lambda\right.
$$

where the last equality comes from the definition of $\Phi\left(X_{T}, c\right)$. Thus the BSDE for $u$ becomes

$$
M_{t}^{u}=e^{-R} e^{\Phi\left(X_{T}, \hat{c}\right)}-\int_{t}^{T} u_{s} M_{s}^{u} d B_{s}
$$

where, since $E\left[M_{T}^{u}\right]=1$, we have

$$
E\left[e^{\Phi\left(X_{T}, \lambda\right)}\right]=e^{R}
$$

so that the IR constraint is satisfied.

\subsection{Examples}

Example 3.1 Suppose the principal is risk-neutral while the agent is risk averse with

$$
U_{2}(x)=x, \quad U_{1}(x)=\log x
$$

Also assume $\sigma_{t}>0$ is a given process and

$$
v_{t}=\sigma_{t} X_{t}, X_{0}=x>0
$$

so that $X_{t}>0$ for all $t$. Moreover, assume that

$$
\tilde{\lambda} \triangleq 2 e^{R}-x>0
$$

The first order condition (3.14) gives

$$
C_{T}=\frac{1}{2}\left(X_{T}+\lambda\right)
$$


and in order to satisfy the IR constraint

$$
e^{R}=E\left[C_{T}\right]=\frac{1}{2}(x+\lambda)
$$

we need to take $\lambda=\tilde{\lambda}$. By the assumptions, we have $C_{T}>0$, and $C_{T}$ is then the optimal contract and it is linear. The optimal $u$ is obtained by solving BSDE

$$
\bar{Y}_{t}=E_{t}\left[C_{T}\right]=e^{R}+\int_{0}^{t} \bar{Y}_{t} u_{t} d B_{t}
$$

Noting that

$$
E_{t}\left[C_{T}\right]=\frac{1}{2}\left(X_{t}+\lambda\right)=e^{R}+\int_{0}^{t} \sigma_{t} X_{t} d B_{t}
$$

we get

$$
u_{t}=2 \sigma_{t} \frac{X_{t}}{X_{t}+\lambda}
$$

Since $\lambda>0$, we see that the effort goes down as the output decreases, and goes up when the output goes up. Thus, the incentive effect coming from the fact that the agent is paid an increasing function of the output at the end, translates into earlier times, so when the promise of the future payment gets higher, the agent works harder. Also notice that the effort is bounded in this example (by $2 \sigma_{t}$ ).

Assume now that $\sigma$ is deterministic. The principal's expected utility can be computed to be equal to

$$
E^{u}\left[X_{T}-C_{T}\right]=e^{-R} E\left[C_{T}\left(X_{T}-C_{T}\right)\right]=x-e^{R}+e^{-R} \frac{x^{2}}{4}\left[e^{\int_{0}^{T} \sigma_{s}^{2} d s}-1\right]
$$

The first term, $x-e^{R}$, is what the principal can get if he pays a constant payoff $C_{T}$, in which case the agent would choose $u \equiv 0$. The second term is the extra benefit of inducing the agent to apply non-zero effort. The extra benefit increases quadratically with the initial output, increases exponentially with the volatility squared, and decreases exponentially with the agent's reservation utility. While the principal would like best to have the agent with the lowest $R$, the cost of hiring expensive agents is somewhat offset when the volatility is high (which is not surprising, given that the principal is risk-neutral).

For comparison, we look now at the first-best case in this example. Interestingly, we have

Remark 3.3 Assume that $\sigma_{t}>0$ is deterministic and bounded. Then the principal's firstbest optimal utility is $\infty$.

Proof. We see from (3.15) that, whenever the principal is risk-neutral, a candidate for an optimal contract is a constant contract $C_{T}$. With log-utility for the agent, we set

$$
C_{T}=\lambda
$$


where $\lambda$ is obtained from the IR constraint, and the optimal utility of the principal is obtained from

$$
\sup _{u} E\left[X_{T}-\lambda\right]=\sup _{u}\left[E\left\{x e^{\int_{0}^{T}\left[u_{t} \sigma_{t}-\frac{1}{2} \sigma_{t}^{2}\right] d t+\int_{0}^{T} \sigma_{t} d B_{t}}\right\}-e^{R} e^{E\left\{\int_{0}^{T} \frac{1}{2} u_{t}^{2} d t\right\}}\right] .
$$

Under the assumption that $\sigma$ is deterministic and bounded, we show now that the right side of (3.16) is $\infty$. In fact, for any $n$, set

$$
A_{n} \triangleq\left\{\int_{0}^{\frac{T}{2}} \sigma_{t} d B_{t}>n\right\} \in \mathcal{F}_{\frac{T}{2}} ; \quad \alpha_{n} \triangleq P\left(A_{n}\right) \rightarrow 0 ;
$$

and

$$
u_{t}^{n}(\omega) \triangleq\left\{\begin{array}{l}
\alpha_{n}^{-\frac{1}{2}}, \quad \frac{T}{2} \leq t \leq T, \omega \in A_{n} \\
0, \quad \text { otherwise }
\end{array}\right.
$$

Then the cost is finite:

$$
E\left\{\int_{0}^{T} \frac{1}{2}\left(u_{t}^{n}\right)^{2} d t\right\}=\frac{T}{4} .
$$

However, for a generic constant $c>0$,

$$
\begin{aligned}
& E\left\{x \exp \left(\int_{0}^{T}\left[u_{t}^{n} \sigma_{t}-\frac{1}{2} \sigma_{t}^{2}\right] d t+\int_{0}^{T} \sigma_{t} d B_{t}\right)\right\} \\
& =E\left\{x \exp \left(\alpha_{n}^{-\frac{1}{2}} \int_{\frac{T}{2}}^{T} \sigma_{t} d t \mathbf{1}_{A_{n}}-\int_{0}^{T} \frac{1}{2} \sigma_{t}^{2} d t+\int_{0}^{T} \sigma_{t} d B_{t}\right)\right\} \\
& \geq E\left\{x \exp \left(\alpha_{n}^{-\frac{1}{2}} \int_{\frac{T}{2}}^{T} \sigma_{t} d t-\int_{0}^{T} \frac{1}{2} \sigma_{t}^{2} d t+\int_{0}^{T} \sigma_{t} d B_{t}\right) \mathbf{1}_{A_{n}}\right\} \\
& =E\left\{x \exp \left(\alpha_{n}^{-\frac{1}{2}} \int_{\frac{T}{2}}^{T} \sigma_{t} d t-\int_{0}^{\frac{T}{2}} \frac{1}{2} \sigma_{t}^{2} d t+\int_{0}^{\frac{T}{2}} \sigma_{t} d B_{t}\right) \mathbf{1}_{A_{n}}\right\} \\
& \geq c E\left\{x \exp \left(\alpha_{n}^{-\frac{1}{2}} \int_{\frac{T}{2}}^{T} \sigma_{t} d t+n\right) \mathbf{1}_{A_{n}}\right\} \\
& =c x \exp \left(\alpha_{n}^{-\frac{1}{2}} \int_{\frac{T}{2}}^{T} \sigma_{t} d t+n\right) P\left(A_{n}\right) \\
& =c x \exp \left(\alpha_{n}^{-\frac{1}{2}} \int_{\frac{T}{2}}^{T} \sigma_{t} d t+n\right) \alpha_{n} \geq c x \alpha_{n} e^{c \alpha_{n}^{-\frac{1}{2}}},
\end{aligned}
$$

which obviously diverges to $\infty$ as $\alpha_{n} \rightarrow 0$.

We note that another completely solvable example in this special framework is the case of both the principal and the agent having linear utilities. But in that case it is easily shown that the first-best and the second-best are the same, so there is no need to consider the second-best.

The property that $u_{t}$ is increasing in the output values $X_{t}$ holds true in more general cases. We have the following result whose proof is postponed to the Appendix. 
Proposition 3.3 Suppose that $U_{2}(x)=x$ and $v_{t}=\sigma_{t} X_{t}$ for deterministic $\sigma_{t} \geq 0$. Assume $\lambda$ is non-negative and (3.13) admits an interior solution. Assume further that

$$
\max \left(H_{1}(x), H_{2}(x)\right) \geq 0,
$$

for any $x$ in the domain of $U_{1}$, where

$$
H_{1} \triangleq U_{1}^{\prime} U_{1}^{\prime \prime \prime}+\left(U_{1}^{\prime}\right)^{2} U_{1}^{\prime \prime}-3\left(U_{1}^{\prime \prime}\right)^{2} ; \quad H_{2} \triangleq\left(U_{1}^{\prime}\right)^{4}+U_{1}^{\prime} U_{1}^{\prime \prime \prime}-\left(U_{1}^{\prime}\right)^{2} U_{1}^{\prime \prime}-2\left(U_{1}^{\prime \prime}\right)^{2}+x U_{1}^{\prime} H_{1} .
$$

Then $u_{t}=u\left(t, X_{t}\right)$ for some deterministic function $u$ and $u_{x} \geq 0$.

Remark 3.4 The following examples satisfy the condition (3.18).

(i) $U_{1}(x)=x$. Then

$$
U_{1}^{\prime}=1, U_{1}^{\prime \prime}=U_{1}^{\prime \prime \prime}=0
$$

Thus

$$
H_{1}(x)=0
$$

(ii) $U_{1}(x)=\log (x)$. Then

$$
U_{1}^{\prime}=x^{-1}, U_{1}^{\prime \prime}=-x^{-2}, U_{1}^{\prime \prime \prime}=2 x^{-3} .
$$

Thus

$$
H_{2}(x)=0 .
$$

(iii) $U_{1}(x)=\frac{1}{\gamma} x^{\gamma}$ where $0<\gamma<1$. Then

$$
U_{1}^{\prime}=x^{\gamma-1}, U_{1}^{\prime \prime}=(\gamma-1) x^{\gamma-2}, U_{1}^{\prime \prime \prime}=(\gamma-1)(\gamma-2) x^{\gamma-3} .
$$

Thus

$$
H_{2}(x)=\gamma x^{2 \gamma-4}\left[x^{2 \gamma}+2(1-\gamma) x^{\gamma}+(1-\gamma)\right] \geq 0
$$

Exponential utility $U_{1}(x)=-\frac{1}{\gamma} e^{-\gamma x}$ does not satisfy (3.18).

\section{General case: Necessary conditions}

In the rest of the paper we find necessary conditions, and discuss a possibility of finding sufficient conditions for optimal contracts in a very general framework. This material is technical, and much less intuitive than the special case discussed in the previous section. We use the method of the so-called Stochastic Maximum Principle, as described in the book Yong and Zhou (1999). It is an extension of the Pontryagin maximum principle to the stochastic case. We believe this is the right method to use for the general theory. For example, it naturally leads to the use of remaining expected utility of the agent as the variable on which the solution heavily depends, and also it shows immediately what other variables are important, without having to guess what they should be, as in other approaches. 


\subsection{Standing assumptions}

Recall the setup from Section 2. We need the following assumptions.

(A1.) Function $g:[0, T] \times \mathbb{R} \times \mathbb{R} \times \mathbb{R} \times \Omega \rightarrow \mathbb{R}$ is continuously differentiable with respect to $x, u, v, g_{x}$ is uniformly bounded, and $g_{u}, g_{v}$ have uniform linear growth in $x, u, v$. In addition, $g$ is jointly convex in $(x, u, v), g_{u}>0$ and $g_{u u}>0$.

(A2.) (i) Functions $U_{1}: \mathbb{R}^{2} \rightarrow \mathbb{R}, U_{2}: \mathbb{R} \rightarrow \mathbb{R}$ are differentiable, with $\partial_{1} U_{1}>0, \partial_{2} U_{1}<$ $0, U_{2}^{\prime}>0, U_{1}$ is jointly concave and $U_{2}$ is concave.

(ii) Sometimes we will also need $U_{1} \leq K$ for some constant $K$.

For any $p \geq 1$, denote

$$
L_{T}^{p}\left(Q^{u}\right) \triangleq\left\{\xi \in \mathcal{F}_{T}^{B}: E^{u}\left\{|\xi|^{p}\right\}<\infty\right\} ; \quad L^{p}\left(Q^{u}\right) \triangleq\left\{\eta \in \mathbf{F}^{B}: E^{u}\left\{\int_{0}^{T}\left|\eta_{t}\right|^{p} d t\right\}<\infty\right\},
$$

and define $L_{T}^{p}(Q), L^{p}(Q)$ in a similar way.

We next define the admissible set for the agent's controls.

(A3.) Given a contract $\left(C_{T}, v\right)$, the admissible set $\mathcal{A}\left(C_{T}, v\right)$ of agent's controls associated with this contract is the set of all those $u \in \mathbf{F}^{B}$ such that

(i) Girsanov Theorem holds true for $\left(B^{u}, Q^{u}\right)$;

(ii) $U_{1}\left(C_{T}, G_{T}\right), \partial_{2} U_{1}\left(C_{T}, G_{T}\right) \in L_{T}^{2}\left(Q^{u}\right)$;

(iii) For any bounded $\Delta u \in \mathbf{F}^{B}$, there exists $\varepsilon_{0}>0$ such that for any $\varepsilon \in\left[0, \varepsilon_{0}\right), u^{\varepsilon}$ satisfies (i) and (ii) at above and $\left|u^{\varepsilon}\right|^{4},\left|g^{\varepsilon}\right|^{4},\left|g_{u}^{\varepsilon}\right|^{4},\left|M_{T}^{\varepsilon}\right|^{4}, U_{1}^{2}\left(C_{T}, G_{T}^{\varepsilon}\right),\left|\partial_{2} U_{1}\left(C_{T}, G_{T}^{\varepsilon}\right)\right|^{2}$ are uniformly integrable in $L^{1}(Q)$ or $L_{T}^{1}(Q)$, where

$$
u^{\varepsilon} \triangleq u+\varepsilon \Delta u, \quad G_{T}^{\varepsilon} \triangleq \int_{0}^{T} g^{\varepsilon}(t) d t, \quad M_{t}^{\varepsilon} \triangleq M_{t}^{u^{\varepsilon}}, \quad V_{1}^{\varepsilon} \triangleq V_{1}\left(u^{\varepsilon}\right),
$$

and

$$
g^{\varepsilon}(t) \triangleq g\left(t, X_{t}, u_{t}^{\varepsilon}, v_{t}\right), \quad g_{u}^{\varepsilon}(t) \triangleq g_{u}\left(t, X_{t}, u_{t}^{\varepsilon}, v_{t}\right)
$$

When $\varepsilon=0$ we omit the superscript "0". We note that, for any $u \in \mathcal{A}\left(C_{T}, v\right)$ and $\Delta u, \varepsilon_{0}$ satisfying (A3) (iii), we have $u^{\varepsilon} \in \mathcal{A}\left(C_{T}, v\right)$ for any $\varepsilon \in\left[0, \varepsilon_{0}\right)$. We note also that, under mild assumptions on $\left(C_{T}, v\right)$, all bounded $u$ belong to $\mathcal{A}\left(C_{T}, v\right)$.

The admissible set for the contracts $\left(C_{T}, v\right)$ is more involved. We postpone its description until later. 


\subsection{Necessary Conditions}

\subsubsection{The Agent's problem}

We fix now a contract $\left(C_{T}, v\right), u \in \mathcal{A}\left(C_{T}, v\right)$, and $\Delta u \in \mathbf{F}^{B}$ bounded. Denote, omitting arguments of $U_{1}, \partial_{2} U_{1}$, the "variations"

$$
\begin{aligned}
& \nabla g(t) \triangleq g_{u}\left(t, X_{t}, u_{t}, v_{t}\right) \Delta u_{t} ; \\
& \nabla G_{t} \triangleq \int_{0}^{t} \nabla g(s) d s ; \\
& \nabla M_{t} \triangleq M_{t}\left[\int_{0}^{t} \Delta u_{s} d B_{s}-\int_{0}^{t} u_{s} \Delta u_{s} d s\right]=M_{t} \int_{0}^{t} \Delta u_{s} d B_{s}^{u} \\
& \nabla V_{1} \triangleq E\left\{\nabla M_{T} U_{1}+M_{T} \partial_{2} U_{1} \nabla G_{T}\right\} .
\end{aligned}
$$

Moreover, for any bounded $\Delta u \in \mathbf{F}^{B}$ and $\varepsilon \in\left(0, \varepsilon_{0}\right)$ as in (A3)(iii), denote

$$
\nabla g^{\varepsilon}(t) \triangleq \frac{g^{\varepsilon}(t)-g(t)}{\varepsilon} ; \quad \nabla G_{T}^{\varepsilon} \triangleq \frac{G_{T}^{\varepsilon}-G_{T}}{\varepsilon} ; \quad \nabla M_{T}^{\varepsilon} \triangleq \frac{M_{T}^{\varepsilon}-M_{T}}{\varepsilon} ; \quad \nabla V_{1}^{\varepsilon} \triangleq \frac{V_{1}^{\varepsilon}-V_{1}}{\varepsilon} .
$$

For a given payoff $C_{T}$ and cost variable $G_{T}$, introduce the so-called adjoint processes

$$
\left\{\begin{array}{l}
W_{t}^{A}=E_{t}^{u}\left[U_{1}\left(C_{T}, G_{T}\right)\right]=U_{1}\left(C_{T}, G_{T}\right)-\int_{t}^{T} Z_{s}^{A, 1} d B_{s}^{u} \\
Y_{t}^{A}=E_{t}^{u}\left[\partial_{2} U_{1}\left(C_{T}, G_{T}\right)\right]=\partial_{2} U_{1}\left(C_{T}, G_{T}\right)-\int_{t}^{T} Z_{s}^{A, 2} d B_{s}^{u}
\end{array}\right.
$$

where $Z^{A, i}$ are obtained from Lemma 3.1. The first one represents the agent's remaining expected utility, and the second one the agent's remaining expected marginal cost. The latter becomes unimportant when the utility is separable (when $U_{1}(C, G)=U_{1}(C)-G$ then $\left.Y_{t}^{A} \equiv-1\right)$.

Theorem 4.1 Under our standing assumptions, we have the following differentiation result for the value function of the agent:

$$
\lim _{\epsilon \rightarrow 0} \nabla V_{1}^{\varepsilon}=\nabla V_{1}=E^{u}\left\{\int_{0}^{T} \Gamma_{t}^{A} \Delta u_{t} d t\right\}
$$

where

$$
\Gamma_{t}^{A} \triangleq Z_{t}^{A, 1}+g_{u}\left(t, X_{t}, u_{t}, v_{t}\right) Y_{t}^{A}
$$

In particular, the necessary condition for $u$ to be an optimal control is:

$$
Z_{t}^{A, 1}+g_{u}\left(t, X_{t}, u_{t}, v_{t}\right) Y_{t}^{A} \equiv 0
$$

Proof: See Appendix. 
Remark 4.1 In the separable case the necessary condition becomes $g_{u}=Z^{A, 1}$ (since $Y_{t}^{A} \equiv$ -1). We can interpret $Z^{A, 1}$ as a "derivative" of the agent's remaining utility with respect to the Brownian motion (actually, it is equal to what is called a Malliavin derivative). Thus, at the optimum, in the separable utility case the agent's local marginal cost of effort has to be equal to the sensitivity of the agent's remaining utility with respect to the underlying uncertainty. In the non-separable case, this has to be adjusted by normalizing with the global marginal cost $Y_{t}^{A}$.

We now provide a FBSDE characterization of the necessary condition. We see that given $\left(C_{T}, v\right)$ (and thus also $X$ ), the optimal $u$ should satisfy the FBSDE

$$
\left\{\begin{array}{l}
G_{t}=\int_{0}^{t} g\left(s, X_{s}, u_{s}, v_{s}\right) d s \\
W_{t}^{A}=U_{1}\left(C_{T}, G_{T}\right)-\int_{t}^{T} Z_{s}^{A, 1} d B_{s}^{u} \\
Y_{t}^{A}=\partial_{2} U_{1}\left(C_{T}, G_{T}\right)-\int_{t}^{T} Z_{s}^{A, 2} d B_{s}^{u}
\end{array}\right.
$$

with maximum condition (4.4).

Moreover, since $g_{u u}>0$, we may assume there exists a function $h(t, x, v, z)$ such that

$$
g_{u}(t, x, h(t, x, v, z), v)=z
$$

Note that $\partial_{2} U_{1}<0$, so $Y_{t}^{A}<0$. Thus, (4.4) is equivalent to

$$
u_{t}=h\left(t, X_{t}, v_{t},-Z_{t}^{A, 1} / Y_{t}^{A}\right)
$$

That is, given $\left(C_{T}, v\right)$ and $X$, one may solve the following (self-contained) FBSDE:

$$
\left\{\begin{array}{l}
G_{t}=\int_{0}^{t} g\left(s, X_{s}, h\left(s, X_{s}, v_{s},-Z_{s}^{A, 1} / Y_{s}^{A}\right), v_{s}\right) d s \\
W_{t}^{A}=U_{1}\left(C_{T}, G_{T}\right)+\int_{t}^{T} Z_{s}^{A, 1} h\left(s, X_{s}, v_{s},-Z_{s}^{A, 1} / Y_{s}^{A}\right) d s-\int_{t}^{T} Z_{s}^{A, 1} d B_{s} \\
Y_{t}^{A}=\partial_{2} U_{1}\left(C_{T}, G_{T}\right)+\int_{t}^{T} Z_{s}^{A, 2} h\left(s, X_{s}, v_{s},-Z_{s}^{A, 1} / Y_{s}^{A}\right) d s-\int_{t}^{T} Z_{s}^{A, 2} d B_{s} .
\end{array}\right.
$$

Then, as a necessary condition, the optimal control $u^{C_{T}, v}$ should be defined by (4.7).

\subsubsection{The Principal's problem}

We now characterize the admissible set $\mathcal{A}$ of contracts $\left(C_{T}, v\right)$. Our first requirement is:

(A4.) $\left(C_{T}, v\right)$ is implementable. That is, (4.8) has a unique solution, and $u^{C_{T}, v}$ defined by (4.7) is in $\mathcal{A}\left(C_{T}, v\right)$. 
Note that we found only necessary conditions for the agent. Later we will have some discussion on when the above $u^{C_{T}, v}$ is indeed the agent's optimal control. Thus, solving the principal's problem with $u^{C_{T}, v}$ instead of general $u$ is not quite satisfying the constraint (2.4), but it represents solving a "relaxed" problem over efforts which satisfy the agent's necessary condition of optimality. This is traditionally called "the first-order approach". The approach will produce the optimal solution if the necessary conditions for the agent are also sufficient.

Now, an implementable contract $\left(C_{T}, v\right)$ uniquely determines $u^{C_{T}, v}$. In fact, for fixed $v$, the correspondence between $C_{T}$ and $u^{C_{T}, v}$ is one to one, up to a constant. To see this, we fix some $(u, v)$ and want to find some $C_{T}$ such that $u^{C_{T}, v}=u$. For notational convenience, we denote

$$
Z^{A} \triangleq Z^{A, 2}
$$

If $u=u^{C_{T}, v}$ for some $C_{T}$, then (4.4) holds true for $u$. That is,

$$
Z_{t}^{A, 1}=-g_{u}\left(t, X_{t}, u_{t}, v_{t}\right) Y_{t}^{A} .
$$

Denote by $\tilde{R}$ the agent's expected utility, that is $\tilde{R} \triangleq W_{0}^{A}$. Then (4.5) becomes

$$
\left\{\begin{array}{l}
G_{t}=\int_{0}^{t} g\left(s, X_{s}, u_{s}, v_{s}\right) d s \\
W_{t}^{A}=\tilde{R}-\int_{0}^{t} g_{u}\left(s, X_{s}, u_{s}, v_{s}\right) Y_{s}^{A} d B_{s}^{u} ; \\
Y_{t}^{A}=\partial_{2} U_{1}\left(C_{T}, G_{T}\right)-\int_{t}^{T} Z_{s}^{A} d B_{s}^{u} ;
\end{array}\right.
$$

where

$$
W_{T}^{A}=U_{1}\left(C_{T}, G_{T}\right) .
$$

Since $\partial_{1} U_{1}>0$, we may assume there exists a function $H(x, y)$ such that

$$
U_{1}(H(x, y), y)=x
$$

Then (4.10) leads to

$$
C_{T} \triangleq H\left(W_{T}^{A}, G_{T}\right)
$$

Plugging this into (4.9), we get

$$
\left\{\begin{array}{l}
X_{t}=x+\int_{0}^{t} v_{s} d B_{s} \\
G_{t}=\int_{0}^{t} g\left(s, X_{s}, u_{s}, v_{s}\right) d s \\
W_{t}^{A}=\tilde{R}-\int_{0}^{t} g_{u}\left(s, X_{s}, u_{s}, v_{s}\right) Y_{s}^{A} d B_{s}^{u} \\
Y_{t}^{A}=\partial_{2} U_{1}\left(H\left(W_{T}^{A}, G_{T}\right), G_{T}\right)-\int_{t}^{T} Z_{s}^{A} d B_{s}^{u}
\end{array}\right.
$$


Now fix $(\tilde{R}, u, v)$. If FBSDE (4.13) is well-posed, we may define $C_{T}$ by (4.12) and we can easily see that $u^{C_{T}, v}=u$. In this sense, for technical convenience, from now on we consider $(\tilde{R}, u, v)$ (instead of $\left.\left(C_{T}, v\right)\right)$ as a contract, or say, as the principal's control, and we call $u$ the principal's target action. Then (A4) should be rewritten as

(A4'.) We assume that $(\tilde{R}, u, v)$ is an implementable contract, by which we mean:

(i) FBSDE (4.13) is well-posed;

(ii) For $C_{T}$ defined by (4.12), $\left(C_{T}, v\right)$ is implementable in the sense of $(\mathbf{A} 4)$.

We note that the theory of FBSDEs is far from complete. The well-posedness of (4.13) is in general unclear (unless we put strict conditions), In fact, even for linear FBSDEs there is no general result like Lemma 3.1. Instead of adopting too strong technical conditions, in this paper we assume the well-posedness of the involved FBSDEs directly and leave the general FBSDE theory for future research. However, in the separable utility case, the corresponding FBSDEs will become decoupled FBSDEs and thus we can use Lemma 3.1 to establish their well-posedness, as we will see later.

Now for any $(u, v)$ and any bounded $(\Delta u, \Delta v)$, denote

$$
\begin{aligned}
& u_{t}^{\varepsilon} \triangleq u_{t}+\varepsilon \Delta u_{t} ; \quad v_{t}^{\varepsilon} \triangleq v_{t}+\varepsilon \Delta v_{t} ; \\
& X_{t}^{\varepsilon} \triangleq x+\int_{0}^{t} v_{s}^{\varepsilon} d B_{s} ; \quad G_{T}^{\varepsilon} \triangleq \int_{0}^{T} g\left(t, X_{t}^{\varepsilon}, u_{t}^{\varepsilon}, v_{t}^{\varepsilon}\right) d t ; \\
& \nabla X^{\varepsilon} \triangleq \frac{X^{\varepsilon}-X}{\varepsilon} ; \quad \nabla G_{T}^{\varepsilon} \triangleq \frac{G_{T}^{\varepsilon}-G_{T}}{\varepsilon} ; \quad \nabla V_{2}^{\varepsilon} \triangleq \frac{V_{2}^{\varepsilon}-V_{2}}{\varepsilon} .
\end{aligned}
$$

Denote also with superscript ${ }^{\varepsilon}$ all corresponding quantities.

(A5.) The principal's admissible set $\mathcal{A}$ of controls is the set of all those contracts $(\tilde{R}, u, v)$ such that, for any bounded $(\Delta u, \Delta v)$, there exists a constant $\varepsilon_{1}>0$ such that for for any $\varepsilon \in\left[0, \varepsilon_{1}\right):$

(i) $\left(\mathbf{A} 4^{\prime}\right)$ holds true for $\left(\tilde{R}, u^{\varepsilon}, v^{\varepsilon}\right)$;

(ii) The FBSDEs (4.17) and (4.19) below are well-posed for $\left(\tilde{R}, u^{\varepsilon}, v^{\varepsilon}\right)$;

(iii) $\lim _{\varepsilon \rightarrow 0} \nabla V_{2}^{\varepsilon}=\nabla Y_{0}^{P}$ for $\nabla Y_{0}^{P}$ defined in (4.17) below.

Note again that we will specify sufficient conditions for (A5) in the separable utility case later on. We also assume that $\mathcal{A}$ is not empty.

We now derive the necessary conditions for the Principal's problem. Since

$$
\tilde{R}=E^{u}\left\{W_{T}^{A}\right\}=E^{u}\left\{U_{1}\left(C_{T}, G_{T}\right)\right\}
$$

is the optimal utility of the agent, the condition (2.6) becomes equivalent to

$$
\tilde{R} \geq R
$$

Intuitively it is obvious that the principal would choose $\tilde{R}=R$ in order to maximize her utility. Again, due to the lack of satisfactory theory of FBSDEs, here we simply assume that 
the optimal $\tilde{R}=R$, and we will prove it rigorously in the separable utility case by using the comparison theorem of BSDEs, as we did in the special case.

Given $(u, v)$, let $\left(X, G, W^{A}, Y^{A}, Z^{A}\right)$ be the solution to (4.13) with $\tilde{R}=R$. Define $C_{T}$ by (4.12). This will guarantee that the agent's necessary condition is satisfied. Introduce the principal's remaining expected utility

$$
Y_{t}^{P}=E_{t}^{u}\left[U_{2}\left(X_{T}-C_{T}\right)\right]=U_{2}\left(X_{T}-C_{T}\right)-\int_{t}^{T} Z_{s}^{P} d B_{s}^{u}
$$

By Lemma 3.1 (4.15) is well-posed. Then the principal's problem is to choose optimal $(u, v)$ in order to maximize

$$
V_{2}(u, v) \triangleq E^{u}\left\{Y_{T}^{P}\right\}=Y_{0}^{P}
$$

Similarly as before, denote, omitting functions' arguments,

$$
\begin{aligned}
& \nabla X_{t}=\int_{0}^{t} \Delta v_{s} d B_{s} \\
& \nabla g_{u}=g_{u u} \Delta u+g_{u v} \Delta v+g_{u x} \nabla X \\
& \nabla G_{T}=\int_{0}^{T}\left[g_{x} \nabla X_{t}+g_{u} \Delta u_{t}+g_{v} \Delta v_{t}\right] d t .
\end{aligned}
$$

Moreover, consider the following FBSDE system

$$
\left\{\begin{array}{l}
\nabla W_{t}^{A}=\int_{0}^{t} g_{u} Y_{s}^{A} \Delta u_{s} d s-\int_{0}^{t}\left[g_{u} \nabla Y_{s}^{A}+Y_{s}^{A} \nabla g_{u}\right] d B_{s}^{u} \\
\nabla Y_{t}^{A}=\partial_{12} U_{1} \nabla C_{T}+\partial_{22} U_{1} \nabla G_{T}+\int_{t}^{T} Z_{s}^{A} \Delta u_{s} d s-\int_{t}^{T} \nabla Z_{s}^{A} d B_{s}^{u} \\
\nabla Y_{t}^{P}=U_{2}^{\prime}\left[\nabla X_{T}-\nabla C_{T}\right]+\int_{t}^{T} Z_{s}^{P} \Delta u_{s} d s-\int_{t}^{T} \nabla Z_{s}^{P} d B_{s}^{u} .
\end{array}\right.
$$

where $\nabla C_{T}$ is defined by

$$
\nabla W_{T}^{A}=\partial_{1} U_{1} \nabla C_{T}+\partial_{2} U_{1} \nabla G_{T} ;
$$

For the general framework we have here, we need to introduce the following "adjoint processes" $X_{i}, Y_{i}, Z_{i}$, which, unfortunately, do not all have a direct economic intuition:

$$
\left\{\begin{aligned}
X_{t}^{1} & =\int_{0}^{t} g_{u} Z_{s}^{1} d s \\
X_{t}^{2} & =\int_{0}^{t}\left[g_{u x} Z_{s}^{1} Y_{s}^{A}+g_{x} Y_{s}^{2}\right] d s \\
Y_{t}^{1} & =\frac{1}{\partial_{1} U_{1}}\left[U_{2}^{\prime}-X_{T}^{1} \partial_{12} U_{1}\right]-\int_{t}^{T} Z_{s}^{1} d B_{s}^{u} \\
Y_{t}^{2} & =\frac{\partial_{2} U_{1}}{\partial_{1} U_{1}}\left[U_{2}^{\prime}-X_{T}^{1} \partial_{12} U_{1}\right]+X_{T}^{1} \partial_{22} U_{1}-\int_{t}^{T} Z_{s}^{2} d B_{s}^{u} \\
Y_{t}^{3} & =X_{T}^{2}+U_{2}^{\prime}-\int_{t}^{T} Z_{s}^{3} d B_{s}^{u}
\end{aligned}\right.
$$


Theorem 4.2 Under (A5), we have

$$
\nabla Y_{0}^{P}=E^{u}\left\{\int_{0}^{T} \Gamma_{t}^{P, 1} \Delta u_{t} d t+\int_{0}^{T} \Gamma_{t}^{P, 2} \Delta v_{t} d t\right\}
$$

where

$$
\left\{\begin{array}{l}
\Gamma_{t}^{P, 1} \triangleq Z_{t}^{P}-g_{u} Y_{t}^{1} Y_{t}^{A}+X_{t}^{1} Z_{t}^{A}+g_{u u} Z_{t}^{1} Y_{t}^{A}+g_{u} Y_{t}^{2} \\
\Gamma_{t}^{P, 2} \triangleq g_{u v} Z_{t}^{1} Y_{t}^{A}+g_{v} Y_{t}^{2}+Z_{t}^{3}+u_{t}\left(Y_{t}^{3}-X_{t}^{2}\right) .
\end{array}\right.
$$

In particular, the necessary condition for $(u, v)$ to be an optimal control is:

$$
\Gamma_{t}^{P, 1}=\Gamma_{t}^{P, 2}=0
$$

Proof. See Appendix.

In summary, we have the following system of necessary conditions for the principal:

$$
\left\{\begin{aligned}
X_{t} & =x+\int_{0}^{t} v_{s} d B_{s} \\
G_{t} & =\int_{0}^{t} g\left(s, X_{s}, u_{s}, v_{s}\right) d s \\
W_{t}^{A} & =R-\int_{0}^{t} g_{u} Y_{s}^{A} d B_{s}^{u} \\
X_{t}^{1} & =\int_{0}^{t} g_{u} Z_{s}^{1} d s \\
X_{t}^{2} & =\int_{0}^{t}\left[g_{u x} Z_{s}^{1} Y_{s}^{A}+g_{x} Y_{s}^{2}\right] d s ; \\
Y_{t}^{A} & =\partial_{2} U_{1}\left(H\left(W_{T}^{A}, G_{T}\right), G_{T}\right)-\int_{t}^{T} Z_{s}^{A} d B_{s}^{u} \\
Y_{t}^{P} & =U_{2}\left(X_{T}-H\left(W_{T}^{A}, G_{T}\right)\right)-\int_{t}^{T} Z_{s}^{P} d B_{s}^{u} \\
Y_{t}^{1} & =\frac{1}{\partial_{1} U_{1}}\left[U_{2}^{\prime}-X_{T}^{1} \partial_{12} U_{1}\right]-\int_{t}^{T} Z_{s}^{1} d B_{s}^{u} \\
Y_{t}^{2} & =\frac{\partial_{2} U_{1}}{\partial_{1} U_{1}}\left[U_{2}^{\prime}-X_{T}^{1} \partial_{12} U_{1}\right]+X_{T}^{1} \partial_{22} U_{1}-\int_{t}^{T} Z_{s}^{2} d B_{s}^{u} \\
Y_{t}^{3} & =X_{T}^{2}+U_{2}^{\prime}-\int_{t}^{T} Z_{s}^{3} d B_{s} ;
\end{aligned}\right.
$$

with maximum condition (4.22).

In particular, if (4.22) has a unique solution

$$
\begin{aligned}
& u_{t}=h_{1}\left(t, X_{t}, Y_{t}^{1} Y_{t}^{A}, Y_{t}^{2}, Z_{t}^{P}+X_{t}^{1} Z_{t}^{A}, Z_{t}^{1} Y_{t}^{A}, Z_{t}^{3}\right) \\
& v_{t}=h_{2}\left(t, X_{t}, Y_{t}^{1} Y_{t}^{A}, Y_{t}^{2}, Z_{t}^{P}+X_{t}^{1} Z_{t}^{A}, Z_{t}^{1} Y_{t}^{A}, Z_{t}^{3}\right)
\end{aligned}
$$

then, by plugging $\left(h_{1}, h_{2}\right)$ into (4.23) we obtain a self contained FBSDE. 


\subsubsection{Fixed volatility case}

If the principal has no control on $v$, then both $v$ and $X$ are fixed. In this case, along the variation one can only choose $\Delta v=0$. Then (4.23) can be simplified as

$$
\left\{\begin{array}{l}
G_{t}=\int_{0}^{t} g\left(s, X_{s}, u_{s}, v_{s}\right) d s \\
W_{t}^{A}=R-\int_{0}^{t} g_{u} Y_{s}^{A} d B_{s}^{u} \\
X_{t}^{1}=\int_{0}^{t} g_{u} Z_{s}^{1} d s \\
Y_{t}^{A}=\partial_{2} U_{1}\left(H\left(W_{T}^{A}, G_{T}\right), G_{T}\right)-\int_{t}^{T} Z_{s}^{A} d B_{s}^{u} \\
Y_{t}^{P}=U_{2}\left(X_{T}-H\left(W_{T}^{A}, G_{T}\right)\right)-\int_{t}^{T} Z_{s}^{P} d B_{s}^{u} \\
Y_{t}^{1}=\frac{U_{2}^{\prime}-X_{T}^{1} \partial_{12} U_{1}}{\partial_{1} U_{1}}-\int_{t}^{T} Z_{s}^{1} d B_{s}^{u} \\
Y_{t}^{2}=\frac{\partial_{2} U_{1}}{\partial_{1} U_{1}}\left[U_{2}^{\prime}-X_{T}^{1} \partial_{12} U_{1}\right]+X_{T}^{1} \partial_{22} U_{1}-\int_{t}^{T} Z_{s}^{2} d B_{s}^{u}
\end{array}\right.
$$

with maximum condition

$$
\Gamma_{t}^{P, 1} \triangleq Z_{t}^{P}-g_{u} Y_{t}^{1} Y_{t}^{A}+X_{t}^{1} Z_{t}^{A}+g_{u u} Z_{t}^{1} Y_{t}^{A}+g_{u} Y_{t}^{2}=0
$$

\subsection{Separable Utilities}

In this subsection we assume the agent has a separable utility function, namely,

$$
U_{1}\left(C_{T}, G_{T}\right)=U_{1}\left(C_{T}\right)-G_{T}
$$

Here we abuse the notation $U_{1}$. We note that if $U_{1}^{\prime}>0$ and $U_{1}^{\prime \prime} \leq 0$, then Assumption A.2 (i) still holds true.

\subsubsection{The agent's problem}

In this case obviously we have

$$
Y_{t}^{A}=-1 ; \quad Z_{t}^{A, 2}=0
$$

Then (4.3) becomes

$$
\Gamma_{t}^{A} \triangleq Z_{t}^{A, 1}-g_{u}\left(t, X_{t}, u_{t}, v_{t}\right) .
$$

Denote $\tilde{W}_{t}^{A} \triangleq W_{t}^{A}+\int_{0}^{t} g d s$. Then (4.5) and (4.8) become

$$
\tilde{W}_{t}^{A}=U_{1}\left(C_{T}\right)+\int_{t}^{T}\left[u_{s} Z_{s}^{A, 1}-g\right] d s-\int_{t}^{T} Z_{s}^{A, 1} d B_{s} ;
$$


and

$\tilde{W}_{t}^{A}=U_{1}\left(C_{T}\right)+\int_{t}^{T}\left[Z_{s}^{A, 1} h\left(s, X_{s}, v_{s}, Z_{s}^{A, 1}\right)-g\left(s, X_{s}, h\left(s, X_{s}, v_{s}, Z_{s}^{A, 1}\right), v_{s}\right)\right] d s-\int_{t}^{T} Z_{s}^{A, 1} d B_{s}$

respectively.

\subsubsection{The principal's problem}

First one can check straightforwardly that

$$
Y^{A}=-1 ; \quad Z^{A}=0 ; \quad Y^{2}=-Y^{1} ; \quad Z^{2}=-Z^{1} .
$$

Denote

$$
J_{1} \triangleq U_{1}^{-1} ; \quad \tilde{W}_{t}^{A} \triangleq W_{t}^{A}+G_{t} ; \quad \tilde{Y}_{t}^{3} \triangleq Y_{t}^{3}-X_{t}^{2} .
$$

Then (4.12) and (4.21) become,

$$
C_{T}=J_{1}\left(\tilde{W}_{T}^{A}\right) ; \quad \Gamma_{t}^{P, 1} \triangleq Z_{t}^{P}-g_{u u} Z_{t}^{1} ; \quad \Gamma_{t}^{P, 2} \triangleq Z_{t}^{3}+u_{t} \tilde{Y}_{t}^{3}-g_{v} Y_{t}^{1}-g_{u v} Z_{t}^{1}
$$

Therefore, (4.23) becomes

$$
\left\{\begin{array}{l}
X_{t}=x+\int_{0}^{t} v_{s} d B_{s} \\
\tilde{W}_{t}^{A}=R+\int_{0}^{t} g d s+\int_{0}^{t} g_{u} d B_{s}^{u} \\
Y_{t}^{P}=U_{2}\left(X_{T}-J_{1}\left(\tilde{W}_{T}^{A}\right)\right)-\int_{t}^{T} Z_{s}^{P} d B_{s}^{u} \\
Y_{t}^{1}=\frac{U_{2}^{\prime}\left(X_{T}-J_{1}\left(\tilde{W}_{T}^{A}\right)\right)}{U_{1}^{\prime}\left(J_{1}\left(\tilde{W}_{T}^{A}\right)\right)}-\int_{t}^{T} Z_{s}^{1} d B_{s}^{u} \\
\tilde{Y}_{t}^{3}=U_{2}^{\prime}\left(X_{T}-J_{1}\left(\tilde{W}_{T}^{A}\right)\right)-\int_{t}^{T}\left[g_{x} Y_{s}^{1}+g_{u x} Z_{s}^{1}\right] d s-\int_{t}^{T} \tilde{Z}_{s}^{3} d B_{s}^{u}
\end{array}\right.
$$

with maximum conditions $\Gamma_{t}^{P, 1}=\Gamma_{t}^{P, 2}=0$.

As mentioned in $\S 4.2$, we shall specify some sufficient conditions for the well-posedness of the FBSDEs in this case. First, under the integrability conditions in (A5') below, $X$ and $\tilde{W}^{A}$ are well defined. Applying Lemma 3.1 on $\left(Y^{P}, Z^{P}\right),\left(Y^{1}, Z^{1}\right)$ and then on $\left(\tilde{Y}^{3}, \tilde{Z}^{3}\right)$, we see that (4.33) is well-posed. Therefore, FBSDEs (4.9), (4.17), and (4.19) are well-posed in this case.

Recall (4.14) and define other $\varepsilon$-terms similarly. We now modify $\mathcal{A}$ as follows.

(A5'.) The principal's admissible set $\mathcal{A}$ of controls is redefined as the set of all those contracts $(\tilde{R}, u, v)$ such that, for any bounded $(\Delta u, \Delta v)$, there exists a constant $\varepsilon_{1}>0$ such that for any $\varepsilon \in\left[0, \varepsilon_{1}\right)$ : 
(i) $u^{\varepsilon}, v^{\varepsilon}, M_{T}^{\varepsilon},\left[M_{T}^{\varepsilon}\right]^{-1}, g^{\varepsilon}, g_{u}^{\varepsilon}, g_{v}^{\varepsilon}, g_{x}^{\varepsilon}, g_{u u}^{\varepsilon}, g_{u v}^{\varepsilon}, g_{u x}^{\varepsilon}, U_{1}^{\varepsilon}, U_{2}^{\varepsilon},\left[U_{2}^{\prime}\right]^{\varepsilon}$, and $\left[J_{1}^{\prime}\right]^{\varepsilon}$ are uniformly integrable in $L^{p_{0}}(Q)$ or $L_{T}^{p_{0}}(Q)$, for some $p_{0}$ large enough (where $J_{1}=U_{1}^{-1}$ ).

(ii) $u \in \mathcal{A}\left(C_{T}, v\right)$ and $\left(C_{T}, v\right)$ is implementable in the sense of (A4), where $C_{T}$ is defined in $(4.32)$;

Note that we may specify $p_{0}$ as in (A5). But in order to simplify the presentation and to focus on the main ideas, we assume $p_{0}$ is as large as we want.

Theorem 4.3 Assume (A5'). Then (A5) holds true and the optimal $\tilde{R}$ is equal to $R$.

Proof: We first show that the principal's optimal control $\tilde{R}$ is $R$. In fact, for fixed $(u, v)$, let superscript ${ }^{\tilde{R}}$ denote the processes corresponding to $\tilde{R}$. Then obviously $\tilde{W}_{t}^{A, \tilde{R}} \geq \tilde{W}_{t}^{A, R}$ for any $\tilde{R} \geq R$. Since

$$
\partial_{1} J_{1}(x)=\frac{1}{\partial_{1} U_{1}\left(J_{1}(x)\right)}>0, \quad U_{2}^{\prime}>0
$$

we get

$$
Y_{T}^{P, \tilde{R}}=U_{2}\left(X_{T}-C_{T}^{\tilde{R}}\right)=U_{2}\left(X_{T}-J_{1}\left(\tilde{W}_{T}^{A, \tilde{R}}\right)\right) \leq U_{2}\left(X_{T}-J_{1}\left(\tilde{W}_{T}^{A, R}\right)\right)=Y_{T}^{P, R}
$$

Therefore,

$$
Y_{0}^{P, \tilde{R}}=E^{u}\left\{Y_{T}^{P, \tilde{R}}\right\} \leq E^{u}\left\{Y_{T}^{P, R}\right\}=Y_{0}^{P, R} .
$$

Thus, optimal $\tilde{R}$ is equal to $R$.

It remains to prove

$$
\lim _{\varepsilon \rightarrow 0} \nabla V_{2}^{\varepsilon}=\nabla Y_{0}^{P}
$$

We postpone the proof to the Appendix.

To end this subsection, for future use we note that (4.12) becomes

$$
C_{T}=J_{1}\left(R+\int_{0}^{T} g_{u}\left(t, X_{t}, u_{t}, v_{t}\right) d B_{t}^{u}+\int_{0}^{T} g\left(t, X_{t}, u_{t}, v_{t}\right) d t\right) .
$$

This means that the principal's problem is

$$
\begin{aligned}
\sup _{u, v} & E^{u}\left\{U _ { 2 } \left(x+\int_{0}^{T} u_{t} v_{t} d t+\int_{0}^{T} v_{t} d B_{t}^{u}\right.\right. \\
& \left.\left.-J_{1}\left(R+\int_{0}^{T} g_{u}\left(t, X_{t}, u_{t}, v_{t}\right) d B_{t}^{u}+\int_{0}^{T} g\left(t, X_{t}, u_{t}, v_{t}\right) d t\right)\right)\right\} .
\end{aligned}
$$




\subsubsection{Fixed volatility case}

If we also assume $v$ (hence $X$ ) is fixed, then (4.33) becomes

$$
\left\{\begin{array}{l}
\tilde{W}_{t}^{A}=R+\int_{0}^{t} g d s+\int_{0}^{t} g_{u} d B_{s}^{u} \\
Y_{t}^{P}=U_{2}\left(X_{T}-J_{1}\left(\tilde{W}_{T}^{A}\right)\right)-\int_{t}^{T} Z_{s}^{P} d B_{s}^{u} \\
Y_{t}^{1}=\frac{U_{2}^{\prime}\left(X_{T}-J_{1}\left(\tilde{W}_{T}^{A}\right)\right)}{U_{1}^{\prime}\left(J_{1}\left(\tilde{W}_{T}^{A}\right)\right)}-\int_{t}^{T} Z_{s}^{1} d B_{s}^{u}
\end{array}\right.
$$

with maximum condition $\Gamma_{t}^{P, 1}=0$.

\section{General case: Sufficient conditions}

\section{$5.1 \quad$ A general result}

If the necessary condition uniquely determines a candidate for the optimal solution, it is also a sufficient condition, if an optimal solution exists. We here discuss the existence of an optimal solution. In general, our maximization problems are non-concave, so we have to use infinite dimensional non-convex optimization methods.

Let $\mathcal{H}$ be a Hilbert space with norm $\|\cdot\|$ and inner product $<\cdot>$. Let $F: \mathcal{H} \rightarrow \mathbb{R}$ be a functional with Fréchet derivative $f: \mathcal{H} \rightarrow \mathcal{H}$. That is, for any $h, \Delta h \in \mathcal{H}$,

$$
\lim _{\varepsilon \rightarrow 0} \frac{1}{\varepsilon}[F(h+\varepsilon \Delta h)-F(h)]=<f(h), \Delta h>.
$$

The following theorem is a direct consequence of the so-called Ekeland's variational principle, see Ekeland (1974).

Theorem 5.1 Assume

(A1) F is continuous;

(A2) There exists unique $h^{*} \in H$ such that $f\left(h^{*}\right)=0$;

(A3) For $\forall \varepsilon>0, \exists \delta>0$ such that $\left\|F(h)-F\left(h^{*}\right)\right\| \leq \varepsilon$ whenever $\|f(h)\| \leq \delta$.

(A4) $V \triangleq \sup _{h \in H} F(h)<\infty$.

Then $h^{*}$ is the maximum argument of $F$. That is, $F\left(h^{*}\right)=V$.

Remark 5.1 (1) A sufficient condition for (A3) is that $f$ is invertible and $f^{-1}$ is continuous at 0 .

(2) If $\mathcal{H}=\mathbb{R}$ and $f$ is continuous and invertible, then $F$ is either convex or concave, and thus the result obviously holds true.

(3) If (A4) is replaced by $\inf _{h \in H} F(h)>-\infty$, then $h^{*}$ is the minimum argument of $F$. 


\subsection{Sufficient conditions for the principal-agent problem}

\subsubsection{The agent's problem: Separable utility}

Assume that the utility is separable, $U_{1}(C, G)=U_{1}(C)-G$. We have

$$
W_{t}^{A}=E^{Q^{u}}\left[U_{1}\left(C_{T}\right)-\int_{0}^{T} g\left(s, X_{s}, u_{s}, v_{s}\right) d s\right]+\int_{0}^{t} Z_{s}^{A, 1} d B_{s}^{u}
$$

We define $\tilde{W}^{A}=W_{t}^{A}+\int_{0}^{t} g\left(s, X_{s}, u_{s}, v_{s}\right) d s$. Then, switching to Brownian Motion $B$, we have

$$
\tilde{W}_{t}^{A}=U_{1}\left(C_{T}\right)+\int_{t}^{T}\left[u_{s} Z_{s}^{A, 1}-g\left(s, X_{s}, u_{s}, v_{s}\right)\right] d s-\int_{t}^{T} Z_{s}^{A, 1} d B_{s}
$$

Note that $\tilde{W}_{0}^{A}=E^{Q^{u}}\left[U_{1}\left(C_{T}\right)-\int_{0}^{T} g\left(s, X_{s}, u_{s}, v_{s}\right) d s\right]$, so the agent's problem is to maximize $\tilde{W}_{0}^{A}$ over $u$. By the comparison theorem for BSDE's, since $U_{1}\left(C_{T}\right)$ is fixed, from (5.1) we see that $\tilde{W}_{0}^{A}$ will be maximized if the drift $u_{s} Z_{s}^{A, 1}-g\left(s, X_{s}, u_{s}, v_{s}\right)$ is maximized, which is the case if $Z_{s}^{A, 1}=g_{u}\left(s, X_{s}, u_{s}, v_{s}\right)$. This is exactly the necessary condition (4.4), taking into account (4.27). Thus, (4.4) is also a sufficient condition for optimality for the agent's problem.

\subsubsection{The agent's problem: general case}

We now discuss what the conditions of Theorem 5.1 boil down to. Fix $\left(C_{T}, v\right)$, and let $\mathcal{H}$ be the set of all admissible $u$ and

$$
<u^{1}, u^{2}>\triangleq E\left\{\int_{0}^{T} u_{t}^{1} u_{t}^{2} d t\right\} .
$$

For the functional $V_{1}: \mathcal{H} \rightarrow \mathbb{R}$ defined by $V_{1}(u) \triangleq V_{1}\left(u ; C_{T}, v\right)$, by (4.2) $V_{1}$ is Fréchet differentiable with Fréchet derivative $\Gamma^{A}$. More precisely, for any $u$, we solve (4.5) (without assuming (4.4)) and then define $\Gamma^{A}(u)=\Gamma^{A}$ by (4.3). Under technical assumptions, $\Gamma^{A}$ is a mapping from $\mathcal{H} \rightarrow \mathcal{H}$.

Moreover, we need enough assumptions to guarantee that FBSDE (4.5) is well-posed, meaning, in particular, that $V_{1}$ is continuous in $u$, so that (A1) would be true. We further need that FBSDE (4.5) together with maximum condition (4.4), or equivalently FBSDE (4.8), has a unique solution, and denote $u^{*}$ as the control corresponding to this solution. Then (A2) would be true. To ensure (A3), we need to have, for any sequence of $u$,

$$
\left\|\Gamma^{A}(u)\right\| \rightarrow 0 \Rightarrow W_{0}^{A, u} \rightarrow W_{0}^{A, u^{*}} .
$$

Recalling Remark 5.1 (1), we note that (5.3) can be replaced by the following stronger conditions. Assume for any $\Gamma$, FBSDE (4.5) together with condition $\Gamma_{t}^{A}=\Gamma_{t}$ has a unique 
solution and let $u^{\Gamma}$ denote the corresponding $u$. That is, recalling (4.6) and noting again that $\Gamma_{t}^{A}=\Gamma_{t}$,

$$
\left.u_{t}^{\Gamma}=h\left(t, X_{t}, v_{t},\left[\Gamma_{t}-Z_{t}^{A, 1, \Gamma}\right] / Y_{t}^{A, \Gamma}\right]\right),
$$

where $\left(Y^{A, \Gamma}, Z^{A, 1, \Gamma}\right)$ is the solution of the following FBSDE

$$
\left\{\begin{array}{l}
G_{t}^{\Gamma}=\int_{0}^{t} g\left(s, X_{s}, h\left(s, X_{s}, v_{s},\left[\Gamma_{s}-Z_{s}^{A, 1, \Gamma}\right] / Y_{s}^{A, \Gamma}\right), v_{s}\right) d s \\
W_{t}^{A, \Gamma}=U_{1}\left(C_{T}, G_{T}^{\Gamma}\right)+\int_{t}^{T} Z_{s}^{A, 1, \Gamma} h\left(s, X_{s}, v_{s},\left[\Gamma_{s}-Z_{s}^{A, 1, \Gamma}\right] / Y_{s}^{A, \Gamma}\right) d s-\int_{t}^{T} Z_{s}^{A, 1, \Gamma} d B_{s} \\
Y_{t}^{A, \Gamma}=\partial_{2} U_{1}\left(C_{T}, G_{T}^{\Gamma}\right)+\int_{t}^{T} Z_{s}^{A, 2, \Gamma} h\left(s, X_{s}, v_{s},\left[\Gamma_{s}-Z_{s}^{A, 1, \Gamma}\right] / Y_{s}^{A, \Gamma}\right) d s-\int_{t}^{T} Z_{s}^{A, 2, \Gamma} d B_{s} .
\end{array}\right.
$$

We need that the above FBSDE is well-posed. In particular,

$$
V_{1}\left(u^{\Gamma}\right)=W_{0}^{A, \Gamma} \rightarrow W_{0}^{A, 0}=V_{1}\left(u^{*}\right), \quad \text { as } \quad\|\Gamma\| \rightarrow 0 .
$$

Then (A3) holds.

\subsubsection{The principal's problem}

Here, $\mathcal{H}$ is the admissible set of $(u, v)$ with

$$
<\left(u^{1}, v^{1}\right),\left(u^{2}, v^{2}\right)>\triangleq E\left\{\int_{0}^{T}\left[u_{t}^{1} u_{t}^{2}+v_{t}^{1} v_{t}^{2}\right] d t\right\} .
$$

The functional is $V_{2}(u, v)$ defined in (4.16). By (4.20) $V_{2}$ is Fréchet differentiable with Fréchet derivative $\left(\Gamma^{P, 1}(u, v), \Gamma^{P, 2}(u, v)\right)$. As in $\S 4.2 .1$, we need the following:

(i) Considering $(u, v)$ as parameters, FBSDE (4.23) (without assuming (4.22)) is wellposed;

(ii) FBSDE (4.23) together with (4.22) has a unique solution $\left(u^{*}, v^{*}\right)$;

(iii) For any sequence of $(u, v)$,

$$
\left\|\left(\Gamma^{P, 1}(u, v), \Gamma^{P, 2}(u, v)\right)\right\| \rightarrow 0 \Rightarrow Y_{0}^{P, u, v} \rightarrow Y_{0}^{P, u^{*}, v^{*}} .
$$

Then $\left(u^{*}, v^{*}\right)$ is the optimal control for the principal problem.

Similarly, (iii) can be replaced by the following stronger condition:

(iii') For any $\left(\Gamma^{1}, \Gamma^{2}\right)$, FBSDE (4.23) together with condition $\left(\Gamma_{t}^{P, 1}, \Gamma_{t}^{P, 2}\right)=\left(\Gamma_{t}^{1}, \Gamma_{t}^{2}\right)$ is well-posed. In particular,

$$
V_{2}\left(u^{\Gamma^{1}, \Gamma^{2}}, v^{\Gamma^{1}, \Gamma^{2}}\right) \rightarrow V_{2}\left(u^{0,0}, v^{0,0}\right), \quad \text { as } \quad\left\|\left(\Gamma^{1}, \Gamma^{2}\right)\right\| \rightarrow 0 .
$$




\subsubsection{Fixed volatility case}

In this case $v$ is fixed. Set $\mathcal{H}$ to be the admissible set of $u$ with inner product defined by (5.2). The functional is $V_{2}(u)$ with Frechet derivative $\Gamma^{P, 1}(u)$. We need the following:

(i) Considering $u$ as a parameter, FBSDE (4.24) (without assuming (4.25)) is well-posed;

(ii) FBSDE (4.24) together with (4.25) has a unique solution $u^{*}$;

(iii) For any sequence of $u$,

$$
\left\|\Gamma^{P, 1}(u)\right\| \rightarrow 0 \Rightarrow Y_{0}^{P, u} \rightarrow Y_{0}^{P, u^{*}} .
$$

Then $u^{*}$ is the optimal control of the principal's problem.

Similarly, (iii) can be replaced by the following stronger condition:

(iii') For any $\Gamma$, FBSDE (4.24) together with condition $\Gamma_{t}^{P, 1}=\Gamma_{t}$ is well-posed. In particular,

$$
V_{2}\left(u^{\Gamma}\right) \rightarrow V_{2}\left(u^{0}\right), \quad \text { as } \quad\|\Gamma\| \rightarrow 0
$$

\subsection{Extension of the original Holmstrom-Milgrom problem}

We now illustrate the approach above on a well known example. Assume exponential utilities, $U_{1}(x, y)=U_{1}(x-y)=-e^{-\gamma_{1}(x-y)}$ and $U_{2}(x)=-e^{-\gamma_{2} x}$. Here we abuse the notation $U_{1}$ again. In the original Holmstrom-Milgrom (1987) paper, it was also the case that $v$ was fixed as a given constant, but we do not assume that. The results below are obtained in Schattler and Sung (1993) and Sung (1995) using a different approach.

For the exponential utility we have that

$$
\partial_{2} U_{1}(x, c)=\gamma_{1} U_{1}(x, c)
$$

Therefore, we get, for the agent's problem adjoint processes (4.1), that

$$
\gamma_{1} W_{t}^{A}=Y_{t}^{A}, \quad \gamma_{1} Z_{t}^{A, 1}=Z_{t}^{A, 2}
$$

Thus, the necessary condition (4.4) becomes

$$
Z_{t}^{A, 1}=-\gamma_{1} g_{u}\left(t, X_{t}, u_{t}, v_{t}\right) W_{t}^{A}
$$

Therefore, the agent's remaining utility is

$$
W_{t}^{A}=U_{1}\left(C_{T}-G_{T}\right)+\int_{t}^{T} W_{s}^{A} \gamma_{1} g_{u}\left(s, X_{s}, u_{s}, v_{s}\right) d B_{s}^{u}
$$

For the optimal contract we will have $W_{0}^{A}=R$. Therefore, we get

$$
W_{t}^{A}=R \exp \left(-\frac{1}{2} \int_{0}^{t} \gamma_{1}^{2} g_{u}^{2}\left(s, X_{s}, u_{s}, v_{s}\right) d s-\int_{0}^{t} \gamma_{1} g_{u}\left(s, X_{s}, u_{s}, v_{s}\right) d B_{s}^{u}\right)
$$


Let us first show that the condition (5.6) is also sufficient. For any given control $u$, since $W_{t}^{A}$ is positive, we can write

$$
W_{t}^{A}=-e^{-\gamma_{1}\left(C_{T}-G_{T}\right)}-\int_{t}^{T} W_{s}^{A} \tilde{Z}_{s}^{A, 1} d B_{s}^{u}
$$

where $\tilde{Z}^{A, 1}=Z^{A, 1} / W^{A}$. Denote

$$
\tilde{Y}_{t}=W_{t}^{A} e^{\gamma_{1} G_{t}}
$$

We get, by switching to Brownian Motion $B$,

$$
d \tilde{Y}_{t}=-e^{-\gamma_{1} C_{T}}-\int_{t}^{T} \tilde{Z}_{s}^{A, 1} \tilde{Y}_{s} d B_{s}^{u}+\int_{t}^{T}\left[\tilde{Z}_{s}^{A, 1} \tilde{Y}_{s} u_{s}+\gamma_{1} g\left(s, X_{s}, u_{s}, v_{s}\right) \tilde{Y}_{s}\right] d s .
$$

Note that the agent wants to maximize $W_{0}^{A}=\tilde{Y}_{0}$. By the BSDE Comparison Theorem, the latter is maximized if the drift in (5.8) is maximized. We see that this will be true if condition (5.6) is satisfied, which is then a sufficient condition.

Denote

$$
J_{1}(y) \triangleq U_{1}^{-1}(y)=-\log (-y) / \gamma_{1}
$$

The principal's problem is then to maximize

$$
E^{Q^{u}}\left[U_{2}\left(X_{T}-J_{1}\left(W_{T}^{A}(u)\right)-G_{T}\right)\right]
$$

We now impose the assumption (with a slight abuse of notation) that

$$
g(t, x, u, v)=\mu_{t} x+g(t, u, v)
$$

for some deterministic function $\mu_{t}$. Doing integration by parts we get the following representation for the first part of the cost $G_{T}$ :

$$
\int_{0}^{T} \mu_{s} X_{s} d s=X_{T} \int_{0}^{T} \mu_{s} d s-\int_{0}^{T} \int_{0}^{s} \mu_{u} d u\left[u_{s} v_{s} d s+v_{s} d B_{s}^{u}\right]
$$

If we substitute this into $G_{T}=\int_{0}^{T} \mu_{s} X_{s} d s+\int_{0}^{T} g\left(s, u_{s}, v_{s}\right) d s$, and plug the expression for $X_{T}$ and the expression (5.7) for $\tilde{Y}^{A}$ into (5.9), with $U_{2}(x)=-e^{-\gamma_{i} x}$, we get that we need to minimize

$$
\begin{aligned}
& E^{u}\left[\operatorname { e x p } \left(-\gamma_{2}\left[1-\int_{0}^{T} \mu_{s} d s\right]\left[X_{0}+\int_{0}^{T} u_{t} v_{t} d t\right]+\gamma_{2} \gamma_{1} \int_{0}^{T} \frac{g_{u}^{2}\left(s, u_{s}, v_{s}\right)}{2} d s\right.\right. \\
& +\gamma_{2} \int_{0}^{T} g\left(s, u_{s}, v_{s}\right) d s-\gamma_{2} \int_{0}^{T}\left[\int_{0}^{s} \mu_{r} d r\right] u_{s} v_{s} d s-\gamma_{2}\left[1-\int_{0}^{T} \mu_{s} d s\right] \int_{0}^{T} v_{s} d B_{s}^{u} \\
& \left.\left.+\gamma_{2} \int_{0}^{T} g_{u}\left(s, u_{s}, v_{s}\right) d B_{s}^{u}-\gamma_{2} \int_{0}^{T}\left[\int_{0}^{s} \mu_{r} d r\right] v_{s} d B_{s}^{u}\right)\right] .
\end{aligned}
$$


This is a standard stochastic control problem, for which the solution turns out to be deterministic processes $\hat{u}, \hat{v}$ (as can be verified, once the solution is found, by verifying the corresponding Hamilton-Jacobi-Bellman equation). Assuming that $u, v$ are deterministic, the expectation above can be computed by using the fact that

$$
E^{u}\left[\exp \left(\int_{0}^{T} f_{s} d B_{s}^{u}\right)\right]=\exp \left(\frac{1}{2} \int_{0}^{T} f_{s}^{2} d s\right)
$$

for a given square-integrable deterministic function $f$. Then, the minimization can be done inside the integral in the exponent, and boils down to minimizing over $\left(u_{t}, v_{t}\right)$ the expression

$$
\begin{aligned}
& -\left[1-\int_{t}^{T} \mu_{s} d s\right] u_{t} v_{t}+\gamma_{1} \frac{g_{u}^{2}\left(t, u_{t}, v_{t}\right)}{2}+g\left(t, u_{t}, v_{t}\right) \\
& +\frac{\gamma_{2}}{2}\left\{\left[1-\int_{t}^{T} \mu_{s} d s\right] v_{t}-g_{u}\left(t, u_{t}, v_{t}\right)\right\}^{2} .
\end{aligned}
$$

The optimal contract is found from (4.12), as:

$$
C_{T}=G_{T}-\frac{1}{\gamma_{1}} \log \left(-W_{T}^{A}\right)
$$

where $W^{A}$ should be written not in terms of the Brownian Motion $B^{u}$, but in the terms of the process $X$. Since we have

$$
W_{t}^{A}=R \exp \left(-\int_{0}^{t} \gamma_{1}^{2} g_{u}^{2}\left(s, u_{s}, v_{s}\right) / 2 d s+\int_{0}^{t} \gamma_{1} u_{s} g_{u}\left(s, u_{s}, v_{s}\right) d s-\int_{0}^{t} \gamma_{1} g_{u}\left(s, u_{s}, v_{s}\right) d B_{s}\right)
$$

we get that the optimal contract can be written as (assuming optimal $v_{t}$ is strictly positive)

$$
C_{T}=c+\int_{0}^{T} \mu_{s} X_{s} d s+\int_{0}^{T} \frac{g_{u}\left(s, u_{s}, v_{s}\right)}{v_{s}} d X_{s}
$$

for some constant $c$. If $\mu \equiv 0$ and $\frac{g_{u}\left(s, u_{s}, v_{s}\right)}{v_{s}}$ is a constant, then we get a linear contract.

Let us consider the special case of Holmstrom-Milgrom (1987), with

$$
v \equiv 1, \quad g(t, x, u, v)=u^{2} / 2 .
$$

Then (5.13) becomes

$$
-u_{t}+\gamma_{1} u_{t}^{2} / 2+u_{t}^{2} / 2+\frac{\gamma_{2}}{2}\left\{1-u_{t}\right\}^{2} \text {. }
$$

Minimizing this we get constant optimal $u$ of Holmstrom-Milgrom (1987), given by

$$
\hat{u}=\frac{1+\gamma_{2}}{1+\gamma_{1}+\gamma_{2}}
$$

The optimal contract is linear, and given by $C_{T}=a+b X_{T}$, where $b=\hat{u}$ and $a$ is such that the IR constraint is satisfied,

$$
a=-\frac{1}{\gamma_{1}} \log (-R)-b X_{0}+\frac{b^{2} T}{2}\left(\gamma_{1}-1\right) .
$$

Note that in the limit when $\gamma_{i} \rightarrow 0$ we get the case corresponding to the linear utility function $U_{i}(x)=x$. 


\section{Conclusion}

We provide a new solvable case of Principal-Agent problems with general utility functions and hidden action in models driven by Brownian Motion, which leads to nonlinear contracts. We then formulate a general theory leading to the necessary conditions for the optimal solution. However, the question of the existence of an optimal solution remains open. We analyze both the agent and the principal's problem in weak formulation, thus having a consistent framework.

\section{Appendix}

In this appendix we provide the remaining proofs.

\subsection{Proof of Lemma 3.1}

We first assume $\xi$ is bounded. Then $M_{T}^{u} \xi \in L_{T}^{2}(Q)$. Let $(\tilde{Y}, \tilde{Z})$ be the unique solution to the BSDE

$$
\tilde{Y}_{t}=M_{T}^{u} \xi-\int_{t}^{T} \tilde{Z}_{s} d B_{s} .
$$

Define

$$
Y_{t} \triangleq \tilde{Y}_{t}\left[M_{t}^{u}\right]^{-1}, \quad Z_{t} \triangleq\left[\tilde{Z}_{t}-u_{t} \tilde{Y}_{t}\right]\left[M_{t}^{u}\right]^{-1} .
$$

One can check directly that

$$
d Y_{t}=Z_{t} d B_{t}^{u}, \quad Y_{T}=\xi
$$

Moreover,

$$
Y_{t}=E_{t}\left\{M_{T}^{u} \xi\right\}\left[M_{t}^{u}\right]^{-1}=E_{t}^{u}\{\xi\}
$$

which implies that

$$
E^{u}\left\{\sup _{0 \leq t \leq T}\left|Y_{t}\right|^{2}\right\} \leq C E^{u}\left\{|\xi|^{2}\right\}<\infty .
$$

Then one can easily get $Z \in L^{2}\left(Q^{u}\right)$.

In general, assume $\xi_{n}$ are bounded and $E^{u}\left\{\left|\xi_{n} \rightarrow \xi\right|^{2}\right\} \rightarrow 0$. Let $\left(Y^{n}, Z^{n}\right)$ be the solution to BSDE (3.1) with terminal condition $\xi_{n}$. Then

$$
E^{u}\left\{\sup _{0 \leq t \leq T}\left|Y_{t}^{n}-Y_{t}^{m}\right|^{2}+\int_{0}^{T}\left|Z_{t}^{n}-Z_{t}^{m}\right|^{2} d t\right\} \leq C E^{u}\left\{\left|\xi_{n}-\xi_{m}\right|^{2}\right\} \rightarrow 0 .
$$

Therefore, $\left(Y^{n}, Z^{n}\right)$ converges to some $(Y, Z)$ which satisfies (3.1). 


\subsection{Proof of Theorem 3.1}

We first show that

Lemma 7.1 For any $u \in \mathcal{A}_{1}$, we have

$$
E\left\{e^{2 \int_{0}^{T}\left|u_{t}\right|^{2} d t}\right\}<\infty
$$

and thus Girsanov Theorem holds for $u$.

Proof. Denote

$$
\tau_{n} \triangleq \inf \left\{t: \int_{0}^{t}\left|u_{s}\right|^{2} d s+\left|\int_{0}^{t} u_{s} d B_{s}\right|>n\right\} \wedge T
$$

Then $\tau_{n} \uparrow T$. Moreover,

$$
e^{\int_{0}^{\tau_{n}} u_{t} d B_{t}}=M_{\tau_{n}}^{u} e^{\frac{1}{2} \int_{0}^{\tau_{n}}\left|u_{t}\right|^{2} d t} .
$$

Squaring both sides and taking the expectation, we get

$$
E\left\{e^{2 \int_{0}^{\tau_{n}}\left|u_{t}\right|^{2} d t}\right\}=E\left\{\left|M_{\tau_{n}}^{u}\right|^{2} e^{\int_{0}^{\tau_{n}}\left|u_{t}\right|^{2} d t}\right\} \leq\left[E\left\{\left|M_{\tau_{n}}^{u}\right|^{4}\right\}\right]^{\frac{1}{2}}\left[E\left\{e^{2 \int_{0}^{\tau_{n}}\left|u_{t}\right|^{2} d t}\right\}\right]^{\frac{1}{2}} .
$$

Thus

$$
E\left\{e^{2 \int_{0}^{\tau_{n}}\left|u_{t}\right|^{2} d t}\right\} \leq E\left\{\left|M_{\tau_{n}}^{u}\right|^{4}\right\} \leq E\left\{\left|M_{T}^{u}\right|^{4}\right\}<\infty .
$$

Letting $n \rightarrow \infty$ we get (7.1).

We now show that BSDE (3.2) is wellposed and $\hat{u} \in \mathcal{A}_{1}$. First, by Definition 3.2 (i), we can solve the following linear BSDE

$$
\bar{Y}_{t}=e^{U_{1}\left(C_{T}\right)}-\int_{t}^{T} \bar{Z}_{s} d B_{s} .
$$

Define

$$
\hat{u}_{t} \triangleq \frac{\bar{Z}_{t}}{\bar{Y}_{t}}
$$

Then $(\bar{Y}, \hat{u})$ satisfies $(3.2)$.

Since $\bar{Y}_{t}>0$ is continuous, and $E\left\{\int_{0}^{T}\left|\bar{Z}_{t}\right|^{2} d t\right\}<\infty$, we know $\hat{u}$ satisfies Definition 3.1 (i). Moreover, by straightforward calculation (or recall (3.11)) we have

$$
M_{T}^{\hat{u}}=\left[\bar{Y}_{0}\right]^{-1} e^{U_{1}\left(C_{T}\right)} .
$$

Thus, by Definition 3.2 (i),

$$
E\left\{\left|M_{T}^{\hat{u}}\right|^{4}\right\}=\left[\bar{Y}_{0}\right]^{-4} E\left\{e^{4 U_{1}\left(C_{T}\right)}\right\}<\infty .
$$

Therefore, $\hat{u} \in \mathcal{A}_{1}$. 


\subsection{Proof of Proposition 3.3}

Under our assumptions (3.14) becomes

$$
C_{T}+\frac{1}{U_{1}^{\prime}\left(C_{T}\right)}=X_{T}+\lambda
$$

we know $C_{T}=c\left(X_{T}\right)$ for some deterministic function $c$. Then by Remark 3.1 we know $u_{t}=u\left(t, X_{t}\right)$ and (3.5)-(3.6) hold true with $v(t, x)=\sigma_{t} x$. Note that

$$
u_{x}(t, x)=\frac{\sigma_{t}}{F^{2}}\left[F_{x} F+x\left(F F_{x x}-F_{x}^{2}\right)\right](t, x) .
$$

Without loss of generality, we prove the result only at $t=0$.

Denote

$$
X_{t}^{x}=x+\int_{0}^{t} \sigma_{s} X_{s}^{x} d B_{s} ; \quad \nabla X_{t}=1+\int_{0}^{t} \sigma_{s} \nabla X_{s} d B_{s}
$$

Then

$$
\nabla X_{T}=\frac{X_{T}}{x}
$$

and

$$
\begin{aligned}
F(0, x)= & E\left\{e^{U_{1}\left(c\left(X_{T}^{x}\right)\right)}\right\} \\
F_{x}(0, x)= & E\left\{e^{U_{1}\left(c\left(X_{T}^{x}\right)\right)} U_{1}^{\prime}\left(c\left(X_{T}^{x}\right)\right) c^{\prime}\left(X_{T}^{x}\right) \nabla X_{T}\right\} \\
F_{x x}(0, x)= & E\left\{e ^ { U _ { 1 } ( c ( X _ { T } ^ { x } ) ) } \left[\left|U_{1}^{\prime}\left(c\left(X_{T}^{x}\right)\right) c^{\prime}\left(X_{T}^{x}\right) \nabla X_{T}\right|^{2}\right.\right. \\
& \left.\left.+U_{1}^{\prime \prime}\left(c\left(X_{T}^{x}\right)\right)\left|c^{\prime}\left(X_{T}^{x}\right) \nabla X_{T}\right|^{2}+U_{1}^{\prime}\left(c\left(X_{T}^{x}\right)\right) c^{\prime \prime}\left(X_{T}^{x}\right)\left|\nabla X_{T}\right|^{2}\right]\right\} .
\end{aligned}
$$

Thus, by suppressing the variables,

$$
\begin{aligned}
u_{x}(0, x) & =\frac{\sigma_{0}}{F^{2}}\left[F F_{x}+x\left[F F_{x x}-F_{x}^{2}\right]\right](0, x) \\
& \geq \frac{\sigma_{0}}{F^{2}}\left[F E\left\{e^{U_{1}} U_{1}^{\prime} c^{\prime} \nabla X_{T}\right\}+x F E\left\{e^{U_{1}}\left[U_{1}^{\prime \prime}\left|c^{\prime}\right|^{2}+U_{1}^{\prime} c^{\prime \prime}\right]\left|\nabla X_{T}\right|^{2}\right\}\right. \\
& =\frac{\sigma_{0}}{x F} E\left\{e^{U_{1}}\left[U_{1}^{\prime} c^{\prime} X_{T}+\left[U_{1}^{\prime \prime}\left|c^{\prime}\right|^{2}+U_{1}^{\prime} c^{\prime \prime}\right]\left|X_{T}\right|^{2}\right]\right\} .
\end{aligned}
$$

Note that

$$
\begin{aligned}
c^{\prime}(x) & =\frac{\left|U_{1}^{\prime}\right|^{2}}{\left|U_{1}^{\prime}\right|^{2}-U_{1}^{\prime \prime}}>0 \\
c^{\prime \prime}(x) & =\frac{\left|U_{1}^{\prime}\right|^{2} U_{1}^{\prime \prime \prime}-2 U_{1}^{\prime}\left|U_{1}^{\prime \prime}\right|^{2}}{\left[\left|U_{1}^{\prime}\right|^{2}-U_{1}^{\prime \prime}\right]^{2}} c^{\prime}=\frac{U_{1}^{\prime} U_{1}^{\prime \prime \prime}-2\left|U_{1}^{\prime \prime}\right|^{2}}{\left[\left|U_{1}^{\prime}\right|^{2}-U_{1}^{\prime \prime}\right] U_{1}^{\prime}}\left|c^{\prime}\right|^{2} .
\end{aligned}
$$

Then

$$
U_{1}^{\prime \prime}\left|c^{\prime}\right|^{2}+U_{1}^{\prime} c^{\prime \prime}=\frac{\left|U_{1}^{\prime}\right|^{2} U_{1}^{\prime \prime}+U_{1}^{\prime} U_{1}^{\prime \prime \prime}-3\left|U_{1}^{\prime \prime}\right|^{2}}{\left|U_{1}^{\prime}\right|^{2}-U_{1}^{\prime \prime}}\left|c^{\prime}\right|^{2}=\frac{\left|c^{\prime}\right|^{2}}{\left|U_{1}^{\prime}\right|^{2}-U_{1}^{\prime \prime}} H_{1}\left(c\left(X_{T}\right)\right) .
$$


Denote $y=c\left(X_{T}\right)$. If $H_{1}(y) \geq 0$, then, combining the fact that $X_{T}>0$,

$$
U_{1}^{\prime} c^{\prime} X_{T}+\left[U_{1}^{\prime \prime}\left|c^{\prime}\right|^{2}+U_{1}^{\prime} c^{\prime \prime}\right]\left|X_{T}\right|^{2} \geq 0
$$

We now assume $H_{1}(y)<0$, then $H_{2}(y) \geq 0$. Since $\lambda \geq 0$, we have

$$
0<X_{T} \leq X_{T}+\lambda=y+\frac{1}{U_{1}^{\prime}(y)} .
$$

Thus

$$
\begin{aligned}
& U_{1}^{\prime}\left(c\left(X_{T}\right)\right) c^{\prime}\left(X_{T}\right) X_{T}+\left[U_{1}^{\prime \prime}\left(c\left(X_{T}\right)\left|c^{\prime}\left(X_{T}\right)\right|^{2}+U_{1}^{\prime}\left(X_{T}\right) c^{\prime \prime}\left(X_{T}\right)\right]\left|X_{T}\right|^{2}\right. \\
& =U_{1}^{\prime}\left(c\left(X_{T}\right)\right) c^{\prime}\left(X_{T}\right) X_{T}+\frac{H_{1}}{\left|U_{1}^{\prime}\right|^{2}-U_{1}^{\prime \prime}}(y)\left|c^{\prime}\left(X_{T}\right)\right|^{2}\left|X_{T}\right|^{2} \\
& \geq U_{1}^{\prime}\left(c\left(X_{T}\right)\right) c^{\prime}\left(X_{T}\right) X_{T}+\frac{H_{1}}{\left|U_{1}^{\prime}\right|^{2}-U_{1}^{\prime \prime}}(y)\left|c^{\prime}\left(X_{T}\right)\right|^{2} X_{T}\left(X_{T}+\lambda\right) \\
& =\left|c^{\prime}\right|^{2} X_{T}\left[\frac{\left|U_{1}^{\prime}\right|^{2}-U_{1}^{\prime \prime}}{U_{1}^{\prime}}(y)+\frac{H_{1}}{\left|U_{1}^{\prime}\right|^{2}-U_{1}^{\prime \prime}}(y)\left[y+\frac{1}{U_{1}^{\prime}(y)}\right]\right] \\
& =\frac{\left|c^{\prime}\right|^{2} X_{T}}{U_{1}^{\prime}\left[\left|U_{1}^{\prime}\right|^{2}-U_{1}^{\prime \prime}\right]} H_{2}(y) \geq 0 .
\end{aligned}
$$

So in both cases, we have

$$
U_{1}^{\prime} c^{\prime} X_{T}+\left[U_{1}^{\prime \prime}\left|c^{\prime}\right|^{2}+U_{1}^{\prime} c^{\prime \prime}\right]\left|X_{T}\right|^{2} \geq 0
$$

Thus $u_{x}(0, x) \geq 0$.

\subsection{Proof of Theorem 4.1}

By (A3)(iii), one can easily show that

$$
\sup _{\varepsilon \in\left[0, \varepsilon_{0}\right)} E\left\{\int_{0}^{T}\left[\left|g^{\varepsilon}(t)\right|^{4}+\left|g_{u}^{\varepsilon}(t)\right|^{4}\right] d t+\left|G_{T}^{\varepsilon}\right|^{4}+\left|M_{T}^{\varepsilon}\right|^{4}\right\}<\infty
$$

and

$$
\lim _{\epsilon \rightarrow 0} E\left\{\int_{0}^{T}\left[\left|g^{\varepsilon}(t)-g(t)\right|^{4}+\left|g_{u}^{\varepsilon}(t)-g_{u}(t)\right|^{4}\right] d t+\left|G_{T}^{\varepsilon}-G_{T}\right|^{4}+\left|M_{T}^{\varepsilon}-M_{T}\right|^{4}\right\}=0 .
$$

Lemma 7.2 We have

$$
\lim _{\epsilon \rightarrow 0} E\left\{\int_{0}^{T}\left[\left|\nabla g^{\varepsilon}(t)-\nabla g(t)\right|^{4}\right] d t+\left|\nabla G_{T}^{\varepsilon}-\nabla G_{T}\right|^{4}+\left|\nabla M_{T}^{\varepsilon}-\nabla M_{T}\right|^{2}\right\}=0 .
$$


Proof. First, note that $\nabla g^{\varepsilon}(t)=\int_{0}^{1} g_{u}^{\delta \varepsilon}(t) d \delta \Delta u_{t}$. Then

$$
\left|\nabla g^{\varepsilon}(t)-\nabla g(t)\right| \leq\left|\Delta u_{t}\right| \int_{0}^{1}\left|g_{u}^{\delta \varepsilon}(t)-g_{u}(t)\right| d \delta
$$

By (7.3) we get

$$
\lim _{\epsilon \rightarrow 0} E\left\{\int_{0}^{T}\left|\nabla g^{\varepsilon}(t)-\nabla g(t)\right|^{4} d t\right\}=0
$$

Similarly,

$$
\lim _{\epsilon \rightarrow 0} E\left\{\left|\nabla G_{T}^{\varepsilon}-\nabla G_{T}\right|^{4}\right\}=0 .
$$

Second, noting that $M_{T}^{\varepsilon}=\exp \left(\int_{0}^{T} u_{t}^{\varepsilon} d B_{t}-\frac{1}{2} \int_{0}^{T}\left|u_{t}^{\varepsilon}\right|^{2} d t\right)$ we have

$$
\nabla M_{T}^{\varepsilon}=\int_{0}^{1} M_{T}^{\delta \varepsilon}\left[\int_{0}^{T} \Delta u_{t} d B_{t}-\int_{0}^{T}\left(u_{t}+\delta \varepsilon \Delta u_{t}\right) \Delta u_{t} d t\right] d \delta
$$

Then

$$
\begin{aligned}
& E\left\{\left|\nabla M_{T}^{\varepsilon}-\nabla M_{T}\right|^{2}\right\}=E\left\{\mid \int_{0}^{1}\left[\left(M_{T}^{\delta \varepsilon} \int_{0}^{T} \Delta u_{t} d B_{t}-M_{T} \int_{0}^{T} \Delta u_{t} d B_{t}\right)\right.\right. \\
& \left.\left.-\left(M_{T}^{\delta \varepsilon} \int_{0}^{T}\left(u_{t}+\delta \varepsilon \Delta u_{t}\right) \Delta u_{t} d t-M_{T} \int_{0}^{T} u_{t} \Delta u_{t} d s\right)\right]\left.d \delta\right|^{2}\right\} \\
& \leq C \int_{0}^{1} E\left\{\left|M_{T}^{\delta \varepsilon}-M_{T}\right|^{2}\left|\int_{0}^{T} \Delta u_{t} d B_{t}\right|^{2}\right. \\
& +\left|M_{T}^{\delta \varepsilon}-M_{T}\right|^{2}\left|\int_{0}^{T}\left(u_{t}+\delta \varepsilon \Delta u_{t}\right) \Delta u_{t} d t\right|^{2} \\
& \left.\left.+\left|M_{T}\right|^{2} \mid \int_{0}^{T}\left[\left(u_{t}+\delta \varepsilon \Delta u_{t}\right) \Delta u_{t}-u_{t} \Delta u_{t}\right)\right]\left.d t\right|^{2}\right\} d \delta \\
& \leq C \int_{0}^{1}\left[\sqrt{E\left\{\left|M_{T}^{\delta \varepsilon}-M_{T}\right|^{4}\right\}}\right. \\
& \left.+\sqrt{E\left\{\left|M_{T}^{\delta \varepsilon}-M_{T}\right|^{4}\right.}\right\} \sqrt{\int_{0}^{T} E\left\{\left|\left(u_{t}+\delta \varepsilon \Delta u_{t}\right)\right|^{4}\right\} d t} \\
& \left.+E\left\{\left|M_{T}\right|^{2}\right\} \varepsilon^{2}\right] d \delta \text {. }
\end{aligned}
$$

Then by (7.3) and Assumption A3 (iii) we prove the result.

To prove the theorem, we also need the following simple lemma (see, e.g., Cvitanić, Wan and Zhang (2006)):

Lemma 7.3 Assume $W_{t}=\int_{0}^{t} \alpha_{s} d B_{s}+A_{t}$ is a continuous semimartingale, where $B$ is a Brownian motion. Suppose that

1) $\int_{0}^{T}\left|\alpha_{t}\right|^{2} d t<\infty$ a.s.

2) Both $W_{t}$ and $A_{t}$ are uniformly (in $t$ ) integrable.

Then $E\left[W_{T}\right]=E\left[A_{T}\right]$. 
We now show (4.2). The first equality can be written as

$$
\lim _{\epsilon \rightarrow 0} \nabla V_{1}^{\varepsilon}=E\left\{\nabla M_{T} U_{1}+M_{T} \partial_{2} U_{1} \nabla G_{T}\right\} .
$$

Note that we have

$$
\nabla V_{1}^{\varepsilon}=\frac{V_{1}^{\varepsilon}-V_{1}}{\varepsilon}=E\left[\nabla M_{T}^{\varepsilon} U_{1}^{\varepsilon}+M_{T} \frac{U_{1}^{\varepsilon}-U_{1}}{\varepsilon}\right]
$$

As for the limit of the first term on the right-hand side, we can write

$$
\nabla M_{T}^{\varepsilon} U_{1}^{\varepsilon}-\nabla M_{T} U_{1}=\left[\nabla M_{T}^{\varepsilon}-\nabla M_{T}\right] U_{1}+\nabla M_{T}^{\varepsilon}\left[U_{1}^{\varepsilon}-U_{1}\right] .
$$

By Assumption A3 (iii) and the above $L^{2}$ bounds on $\nabla M_{T}^{\varepsilon}$, this is integrable uniformly with respect to $\varepsilon$, so the expected value (under $Q$ ) converges to zero, which is what we need.

As for the limit of the second term in the right side of (7.6), notice that we have

$$
M_{T} \lim _{\epsilon \rightarrow 0} \frac{U_{1}^{\varepsilon}-U_{1}}{\varepsilon}=M_{T} \partial_{2} U_{1} \nabla G_{T} .
$$

We want to prove the uniform integrability again. We note that

$$
\begin{aligned}
\left|\frac{U_{1}^{\varepsilon}-U_{1}}{\varepsilon}\right|= & \left|\int_{0}^{1} \partial_{2} U_{1}\left(C_{T}, G_{T}+\theta\left(G_{T}^{\varepsilon}-G_{T}\right)\right) d \theta\right|\left|\nabla G_{T}^{\varepsilon}\right| \\
& \leq\left\{\left|\partial_{2} U_{1}\left(C_{T}, G_{T}\right)\right|+\left|\partial_{2} U_{1}\left(C_{T}, G_{T}^{\varepsilon}\right)\right|\right\}\left|\nabla G_{T}^{\varepsilon}\right|
\end{aligned}
$$

where the last inequality is due to monotonicity of $\partial_{2} U_{1}$.

Therefore, we get

$$
\left|M_{T} \frac{U_{1}^{\varepsilon}-U_{1}}{\varepsilon}\right| \leq C\left\{\left|\partial_{2} U_{1}\left(C_{T}, G_{T}\right)\right|^{2}+\left|\partial_{2} U_{1}\left(C_{T}, G_{T}^{\varepsilon}\right)\right|^{2}+\left|\nabla G_{T}^{\varepsilon}\right|^{4}+\left|M_{T}\right|^{4}\right\}
$$

Thus, from Assumption A3 (iii), the left-hand side is uniformly integrable, and the expectations of the terms in (7.7) also converge, and we finish the proof of (7.5).

We now want to prove the second equality of (4.2). We have

$$
\begin{aligned}
\nabla V_{1} & =E\left\{\nabla M_{T} U_{1}+M_{T} \partial_{2} U_{1} \nabla G_{T}\right\} \\
& =E\left\{M_{T} U_{1} \int_{0}^{T} \Delta u_{t} d B_{t}^{u}+M_{T} \partial_{2} U_{1} \int_{0}^{T} g_{u} \Delta u_{t} d t\right\} \\
& =E^{u}\left\{W_{T}^{A} \int_{0}^{T} \Delta u_{t} d B_{t}^{u}+Y_{T}^{A} \int_{0}^{T} g_{u} \Delta u_{t} d t\right\} \\
& =E^{u}\left\{\int_{0}^{T} \Gamma_{t}^{A} \Delta u_{t} d t+\int_{0}^{T} \Gamma_{t}^{B} d B_{t}^{u}\right\}
\end{aligned}
$$

where

$$
\Gamma_{t}^{A} \triangleq Z_{t}^{A, 1}+g_{u}\left(t, X_{t}, u_{t}, v_{t}\right) Y_{t}^{A}, \quad \Gamma_{t}^{B} \triangleq W_{t}^{A} \Delta u_{s}+Z_{t}^{A, 1} \int_{0}^{t} \Delta u_{s} d B_{s}^{u}+Z_{t}^{A, 2} \nabla G_{t}
$$


and the last equality is obtained from Itô's rule, and definitions of $Y^{A, i}, Z^{A, i}$. We need to show

$$
E^{u} \int_{0}^{T} \Gamma_{t}^{B} d B_{t}^{u}=0
$$

We want to use Lemma 7.3 in the last two lines of (7.8), with $\alpha=\Gamma^{B}$ and

$$
\begin{gathered}
W_{t}=W_{t}^{A} \int_{0}^{t} \Delta u_{s} d B_{s}^{u}+Y_{t}^{A} \int_{0}^{t} g_{u}(s) \Delta u_{s} d s \\
A_{t}=\int_{0}^{t} \Gamma_{s}^{A} \Delta u_{s} d s
\end{gathered}
$$

From the BSDE theory and our assumptions we have

$$
E^{u}\left\{\sup _{0 \leq t \leq T}\left(\left|W_{t}^{A}\right|^{2}+\left|Y_{t}^{A}\right|^{2}\right)+\int_{0}^{T}\left(\left|Z_{t}^{A, 1}\right|^{2}+\left|Z_{t}^{A, 2}\right|^{2}\right) d t\right\}<\infty .
$$

From this it is easily verified that

$$
\int_{0}^{T}\left|\Gamma_{t}^{B}\right|^{2} d t<\infty
$$

so that condition 1) of the lemma is satisfied. Next, we have

$$
\begin{aligned}
& E^{u}\left\{\sup _{0 \leq t \leq T}\left|W_{t}\right|\right\} \leq C E^{u}\left[\sup _{0 \leq t \leq T}\left\{\left|W_{t}^{A}\right|^{2}+\left|Y_{t}^{A}\right|^{2}+\right\}+\int_{0}^{T}\left|g_{u}(t)\right|^{2} d t\right] \\
& \leq C+C E\left\{M_{T}^{2}+\int_{0}^{T}\left|g_{u}(t)\right|^{4} d t\right\}<\infty
\end{aligned}
$$

thanks to (7.9) and (7.2). Moreover,

$$
\begin{aligned}
& E^{u}\left\{\sup _{0 \leq t \leq T}\left|A_{t}\right|\right\}=E^{u}\left\{\sup _{0 \leq t \leq T}\left|\int_{0}^{t}\left[Z_{s}^{A, 1}+g_{u}(s) Y_{s}^{A}\right] \Delta u_{s} d s\right|\right\} \\
& \leq C E\left\{M_{T} \int_{0}^{T}\left|Z_{t}^{A, 1}+g_{u}(s) Y_{s}^{A}\right| d t\right\} \\
& \leq C E\left\{\left|M_{T}\right|^{4}+\int_{0}^{T}\left[\left|Z_{t}^{A, 1}\right|^{2}+\left|g_{u}(t)\right|^{4}+\left|Y_{t}^{A}\right|^{2}\right] d t\right\}<\infty
\end{aligned}
$$

The last two bounds ensure that condition 2) of the lemma is satisfied, so that the last term in (7.8) is zero, and we finish the proof of (4.2).

Finally, (4.4) follows directly from (4.2) if $u$ is optimal, as $\Delta u_{t}$ is arbitrary. 


\subsection{Proof of Theorem 4.2}

First the necessity of (4.22) is obvious because $(\Delta u, \Delta v)$ is arbitrary.

Note that

$$
\nabla X_{t}=\int_{0}^{t} \Delta v_{s} d B_{s}^{u}+\int_{0}^{t} u_{s} \Delta v_{s} d s .
$$

Applying Lemma 7.3 repeatedly, we have

$$
\begin{aligned}
& E\left\{\left[\partial_{1} U_{1} \nabla C_{T}+\partial_{2} U_{1} \nabla G_{T}\right] Y_{T}^{1}\right\}=E\left\{\nabla W_{T}^{A} Y_{T}^{1}\right\} \\
& =E\left\{\int_{0}^{T} Y_{t}^{1} g_{u} Y_{t}^{A} \Delta u_{t} d t-\int_{0}^{T} Z_{t}^{1}\left[g_{u} \nabla Y_{t}^{A}+Y_{t}^{A} \nabla g_{u}\right] d t\right\} \\
& =E \\
& \quad\left\{\int_{0}^{T} Y_{t}^{1} g_{u} Y_{t}^{A} \Delta u_{t} d t-\int_{0}^{T} Z_{t}^{1} Y_{t}^{A} \nabla g_{u} d t-\int_{0}^{T} X_{t}^{1} Z_{t}^{A} \Delta u_{t} d t\right. \\
& \left.\quad-X_{T}^{1}\left[\partial_{12} U_{1} \nabla C_{T}+\partial_{22} U_{1} \nabla G_{T}\right]\right\},
\end{aligned}
$$

Note that

$$
\partial_{1} U_{1} Y_{T}^{1}+X_{T}^{1} \partial_{12} U_{1}=U_{2}^{\prime} .
$$

Then

$$
\begin{aligned}
E\left\{U_{2}^{\prime} \nabla C_{T}\right\}=E & \left\{\int_{0}^{T} Y_{t}^{1} g_{u} Y_{t}^{A} \Delta u_{t} d t-\int_{0}^{T} Z_{t}^{1} Y_{t}^{A} \nabla g_{u} d t\right. \\
& \left.\left.-\int_{0}^{T} X_{t}^{1} Z_{t}^{A} \Delta u_{t} d t-\left[X_{T}^{1} \partial_{22} U_{1}+Y_{T}^{1} \partial_{2} U_{1}\right] \nabla G_{T}\right]\right\} .
\end{aligned}
$$


Therefore,

$$
\begin{aligned}
\nabla Y_{0}^{P}= & E\left\{U_{2}^{\prime}\left[\nabla X_{T}-\nabla C_{T}\right]+\int_{0}^{T} Z_{t}^{P} \Delta u_{t} d t\right\} \\
= & E\left\{U_{2}^{\prime} \nabla X_{T}+\int_{0}^{T} Z_{t}^{P} \Delta u_{t} d t+\left[X_{T}^{1} \partial_{22} U_{1}+Y_{T}^{1} \partial_{2} U_{1}\right] \nabla G_{T}\right. \\
& \left.-\int_{0}^{T}\left[\left[g_{u} Y_{t}^{1} Y_{t}^{A}-X_{t}^{1} Z_{t}^{A}\right] \Delta u_{t}-Z_{t}^{1} Y_{t}^{A} \nabla g_{u}\right] d t\right\} \\
= & E\left\{\int_{0}^{T}\left[Z_{t}^{P}-g_{u} Y_{t}^{1} Y_{t}^{A}+X_{t}^{1} Z_{t}^{A}+g_{u u} Z_{t}^{1} Y_{t}^{A}+g_{u} Y_{t}^{2}\right] \Delta u_{t} d t\right. \\
& +\int_{0}^{T}\left[g_{u v} Z_{t}^{1} Y_{t}^{A}+g_{v} Y_{t}^{2}\right] \Delta v_{t} d t \\
& \left.+\int_{0}^{T}\left[g_{u x} Z_{t}^{1} Y_{t}^{A}+g_{x} Y_{t}^{2}\right] \nabla X_{t} d t+U_{2}^{\prime} \nabla X_{T}\right\} \\
= & E\left\{\int_{0}^{T}\left[Z_{t}^{P}-g_{u} Y_{t}^{1} Y_{t}^{A}+X_{t}^{1} Z_{t}^{A}+g_{u u} Z_{t}^{1} Y_{t}^{A}+g_{u} Y_{t}^{2}\right] \Delta u_{t} d t\right. \\
& \left.+\int_{0}^{T}\left[g_{u v} Z_{t}^{1} Y_{t}^{A}+g_{v} Y_{t}^{2}\right] \Delta v_{t} d t+Y_{T}^{3} \nabla X_{T}\right\} \\
= & E\left\{\int_{0}^{T}\left[Z_{t}^{P}-g_{u} Y_{t}^{1} Y_{t}^{A}+X_{t}^{1} Z_{t}^{A}+g_{u u} Z_{t}^{1} Y_{t}^{A}+g_{u} Y_{t}^{2}\right] \Delta u_{t} d t\right. \\
& \left.+\int_{0}^{T}\left[g_{u v} Z_{t}^{1} Y_{t}^{A}+g_{v} Y_{t}^{2}+Z_{t}^{3}+\left(Y_{t}^{3}-X_{t}^{2}\right) u_{t}\right] \Delta v_{t} d t\right\} \\
= & E\left\{\int_{0}^{T} \Gamma_{t}^{P, 1} \Delta u_{t} d t+\int_{0}^{T} \Gamma_{t}^{P, 2} \Delta v_{t} d t\right\} .
\end{aligned}
$$

The proof is complete.

\subsection{Proof of Theorem 4.3}

We have already shown that we can set $\tilde{R}=R$. Recall that

$$
\left\{\begin{array}{l}
X_{t}=x+\int_{0}^{t} v_{s} d B_{s} ; \\
M_{t}=\exp \left(\int_{0}^{t} u_{s} d B_{s}-\frac{1}{2} \int_{0}^{t}\left|u_{s}\right|^{2} d s\right) ; \\
\tilde{W}_{t}^{A}=R+\int_{0}^{t} g d s-\int_{0}^{t} u_{s} g_{u} d s+\int_{0}^{t} g_{u} d B_{s} ; \\
Y_{t}^{P}=U_{2}\left(X_{T}-J_{1}\left(\tilde{W}_{T}^{A}\right)\right)+\int_{t}^{T} u_{s} Z_{s}^{P} d s-\int_{t}^{T} Z_{s}^{P} d B_{s}
\end{array}\right.
$$


and that

$$
\left\{\begin{array}{l}
\nabla X_{t}=\int_{0}^{t} \Delta v_{s} d B_{s} \\
\nabla M_{t}=M_{t}\left[\int_{0}^{t} \Delta u_{s} d B_{s}-\int_{0}^{t} u_{s} \Delta u_{s} d s\right] \\
\nabla \varphi=\varphi_{u} \Delta u_{t}+\varphi_{v} \Delta v_{t}+\varphi_{x} \nabla X_{t}, \quad \varphi=g, g_{u} ; \\
\nabla \tilde{W}_{t}^{A}=\int_{0}^{t} \nabla g d s-\int_{0}^{t}\left[g_{u} \Delta u_{s}+u_{s} \nabla g_{u}\right] d s+\int_{0}^{t} \nabla g_{u} d B_{s} \\
\nabla Y_{t}^{P}=U_{2}^{\prime}\left(X_{T}-J_{1}\left(\tilde{W}_{T}^{A}\right)\right)\left[\nabla X_{T}-\frac{\nabla \tilde{W}_{T}^{A}}{U_{1}^{\prime}\left(J_{1}\left(\tilde{W}_{T}^{A}\right)\right)}\right] \\
\quad+\int_{t}^{T}\left[Z_{s}^{P} \Delta u_{s}+u_{s} \nabla Z_{s}^{P}\right] d s-\int_{t}^{T} \nabla Z_{s}^{P} d B_{s}
\end{array}\right.
$$

To prove (4.34), we need the following result. For any random variable $\xi$ and any $p>0$,

$$
\begin{aligned}
E^{u^{\varepsilon}}\left\{|\xi|^{p}\right\} & =E\left\{M_{T}^{u^{\varepsilon}}|\xi|^{p}\right\} \leq \sqrt{E\left\{\left|M_{T}^{u^{\varepsilon}}\right|^{2}\right\}} \sqrt{E\left\{|\xi|^{2 p}\right\}} \leq C \sqrt{E\left\{|\xi|^{2 p}\right\}} \\
E\left\{|\xi|^{p}\right\} & =E^{u^{\varepsilon}}\left\{\left[M_{T}^{u^{\varepsilon}}\right]^{-1}|\xi|^{p}\right\} \leq \sqrt{E^{u^{\varepsilon}}\left\{\left[M_{T}^{u^{\varepsilon}}\right]^{-2}\right\}} \sqrt{E^{u^{\varepsilon}}\left\{|\xi|^{2 p}\right\}} \\
& =\sqrt{E\left\{\left[M_{T}^{u^{\varepsilon}}\right]^{-1}\right\}} \sqrt{E^{u^{\varepsilon}}\left\{|\xi|^{2 p}\right\}} \leq C \sqrt{E^{u^{\varepsilon}}\left\{|\xi|^{2 p}\right\}}
\end{aligned}
$$

Proof of (4.34): In this proof we use a generic constant $p \geq 1$ to denote the powers, which may vary from line to line. We assume all the involved powers are always less than or equal to the $p_{0}$ in (A5').

First, one can easily show that

$\lim _{\varepsilon \rightarrow 0} E\left\{\sup _{0 \leq t \leq T}\left[\left|X_{t}^{\varepsilon}-X_{t}\right|^{p}+\left|M_{t}^{\varepsilon}-M_{t}\right|^{p}+\left|\tilde{W}_{t}^{A}-\tilde{W}_{t}^{A}\right|^{p}\right]+\int_{0}^{T}\left[\left|g^{\varepsilon}-g\right|^{p}+\left|g_{u}^{\varepsilon}-g_{u}\right|^{p}\right] d t\right\}=0$.

Using the arguments in Lemma 3.1 we have

$$
E^{u^{\varepsilon}}\left\{\left[\int_{0}^{T}\left|Z_{t}^{P, \varepsilon}\right|^{2} d t\right]^{p}\right\} \leq C<\infty
$$

which, by applying (7.10) twice, implies that

$$
E^{u}\left\{\left[\int_{0}^{T}\left|Z_{t}^{P, \varepsilon}\right|^{2} d t\right]^{p}\right\} \leq C<\infty .
$$

Note that

$$
\begin{aligned}
Y_{t}^{P, \varepsilon}-Y_{t}^{P} & =U_{2}^{\varepsilon}-U_{2}+\int_{t}^{T}\left[\varepsilon \Delta u_{s} Z_{s}^{P, \varepsilon}+u_{s}\left[Z_{s}^{P, \varepsilon}-Z_{s}^{P}\right]\right] d s-\int_{t}^{T}\left[Z_{s}^{P, \varepsilon}-Z_{s}^{P}\right] d B_{s} \\
& =U_{2}^{\varepsilon}-U_{2}+\varepsilon \int_{t}^{T} \Delta u_{s} Z_{s}^{P, \varepsilon} d s-\int_{t}^{T}\left[Z_{s}^{P, \varepsilon}-Z_{s}^{P}\right] d B_{s}^{u} .
\end{aligned}
$$

Using the arguments in Lemma 3.1 again we get

$$
\lim _{\varepsilon \rightarrow 0} E^{u}\left\{\sup _{0 \leq t \leq T}\left|Y_{t}^{P, \varepsilon}-Y_{t}^{P}\right|^{p}+\left[\int_{0}^{T}\left|Z_{t}^{P, \varepsilon}-Z_{t}^{P}\right|^{2} d t\right]^{p}\right\}=0,
$$


which, together with (7.10), implies that

$$
\lim _{\varepsilon \rightarrow 0} E\left\{\sup _{0 \leq t \leq T}\left|Y_{t}^{P, \varepsilon}-Y_{t}^{P}\right|^{p}+\left[\int_{0}^{T}\left|Z_{t}^{P, \varepsilon}-Z_{t}^{P}\right|^{2} d t\right]^{p}\right\}=0 .
$$

Next, recall (4.14) one can easily show that

$$
\begin{aligned}
& \lim _{\varepsilon \rightarrow 0} E\left\{\sup _{0 \leq t \leq T}\left[\left|\nabla X_{t}^{\varepsilon}-\nabla X_{t}\right|^{p}+\left|\nabla M_{t}^{\varepsilon}-\nabla M_{t}\right|^{p}+\left|\nabla \tilde{W}_{t}^{A}-\nabla \tilde{W}_{t}^{A}\right|^{p}\right]\right. \\
& \left.\quad+\int_{0}^{T}\left[\left|\nabla g^{\varepsilon}-\nabla g\right|^{p}+\left|\nabla g_{u}^{\varepsilon}-\nabla g_{u}\right|^{p}\right] d t\right\}=0 .
\end{aligned}
$$

Then similar to $(7.11)$ one can prove that

$$
\lim _{\varepsilon \rightarrow 0} E\left\{\sup _{0 \leq t \leq T}\left|\nabla Y_{t}^{P, \varepsilon}-\nabla Y_{t}^{P}\right|^{p}\right\}=0
$$

In particular,

$$
\lim _{\varepsilon \rightarrow 0} \nabla V_{2}^{\varepsilon}=\lim _{\varepsilon \rightarrow 0} \nabla Y_{0}^{P, \varepsilon}=\nabla Y_{0}^{P}
$$

The proof is complete.

\section{References}

[1] Abreu, D., Pearce, D., and Stacchetti, E., "Optimal Cartel Equilibria with Imperfect Monitoring", Journal of Economic Theory, 39, 1986, 251-269.

[2] Abreu, D., Pearce, D., and Stacchetti, E., "Toward a Theory of Discounted Repeated Games with Imperfect Monitoring", Econometrica, 58, 1990, 1041-1063.

[3] Biais, B., Mariotti, T., Plantin, G and J.C. Rochet, "Dynamic Security Design: Convergence to Continuous Time and Asset Pricing Implications", Review of Economic Studies 74, 2007, 345390, 2007.

[4] Bolton, P., and M. Dewatripont, Contract Theory. The MIT Press, 2005.

[5] J. Cvitanić, X. Wan and J. Zhang, "First-Best Contracts for Continuous-Time Principal-Agent Problems". Journal of Applied Mathematics and Stochastic Analysis, vol. 2006, Article ID 95203, 27 pages.

[6] P. DeMarzo and Y. Sannikov, "Optimal Security Design and Dynamic Capital Structure in a Continuous-Time Agency Model", J. of Finance, 16, 2006, p. 2681-2724.

[7] J. Detemple, S. Govindaraj, M. Loewenstein, Optimal Contracts and Intertemporal Incentives with Hidden Actions, working paper, Boston University, 2001. 
[8] I. Ekeland, "On the variational principle", J. Math. Anal. Appl. 47 1974, 324-353.

[9] M. Fujisaki, G. Kalliapur, H. Kunita, "Stochastic Differential Equations for the nonlinear filtering problem", Osaka J. Math, 9 1972, 19-40.

[10] Hellwig, M., and K. M. Schmidt, Discrete-Time Approximations of Holmstrom- Milgrom Brownian-Motion Model of Intertemporal Incentive Provision", Econometrica 70, 2002, p. 2225-2264.

[11] Holmstrom, B. "Moral Hazard and Observability", The Bell Journal of Economics, 10, 1979, p. 74-91.

[12] B. Holmstrom and P. Milgrom, "Aggregation and Linearity in the Provision of Intertemporal Incentives", Econometrica 55 1987, 303-328.

[13] J. Hugonnier and R. Kaniel, "Mutual Fund Portfolio Choice in the Presence of Dynamic Flows". To appear in Mathematical Finance, 2008.

[14] D. G. Luenberger, Optimization by Vector Space Methods. Wiley-Interscience, 1997.

[15] J. Ma, and J. Yong, Forward-Backward Stochastic Differential Equations and Their Applications. Lecture Notes in Math. 1702. Springer, Berlin, 1999.

[16] Mirrlees, J. "Notes on Welfare Economics, Information, and Uncertainty" in Balch, McFadden, and Wu, eds., Essays on Economic Behavior under Uncertainty, Amsterdam: North Holland Publishing Co., 1974.

[17] Mirrlees, J. "The Optimal Structure of Incentives and Authority within an Organization", The Bell Journal of Economics, 7, 1976, p. 105-131.

[18] Muller, H., The First-Best Sharing Rule in the Continuous-Time Principal-Agent Problem with Exponential Utility", Journal of Economic Theory, 79, 1998, 276- 280.

[19] Muller, H., Asymptotic Efficiency in Dynamic Principal-Agent Problems", Journal of Economic Theory, 91, 2000, 292-301.

[20] H. Ou-Yang, "Optimal Contracts in a Continuous-Time Delegated Portfolio Management Problem", Review of Financial Studies 16 2003, 173-208.

[21] Phelan, C. and Townsend, R. "Computing Multi-Period, Information-Constrained Optima", Review of Economic Studies, 58, 1991, 853-881.

[22] Sannikov, Y. "A Continuous-Time Version of the Principal-Agent Problem", Review of Economic Studies, forthcoming, 2007. 
[23] H. Schättler and J. Sung, "The First-Order Approach to the Continuous-Time Principal-Agent Problem with Exponential Utility", J. of Economic Theory 611993 , $331-371$.

[24] J. Sung, "Lectures on the theory of contracts in corporate finance", preprint, University of Illinois at Chicago, 2001.

[25] J. Sung, "Linearity with project selection and controllable diffusion rate in continuoustime principal-agent problems", RAND J. of Economics 26 1995, 720-743.

[26] Sung, J., "Optimal Contracts under Adverse Selection and Moral Hazard: a Continuous-Time Approach", Review of Financial Studies 18, 2005, 1021 - 1073.

[27] N. Williams, "On Dynamic Principal-Agent Problems in Continuous Time". Working paper, Princeton University, 2004.

[28] J. Yong, "Completeness of Security Markets and Solvability of Linear Backward Stochastic Differential Equations". Working paper, University of Central Florida, 2004.

[29] J. Yong and X.Y. Zhou, "Stochastic Controls: Hamiltonian Systems and HJB Equations," Springer-Verlag, New York, 1999. 